\title{
TRIADS: a phase-resolving model for nonlinear shoaling of directional wave spectra
}

\author{
Alex Sheremet $^{\mathrm{a}, *}$, Justin R. Davis ${ }^{\mathrm{a}}$, Miao Tian $^{\mathrm{a}}$, Jeffrey L. Hanson ${ }^{\mathrm{b}}$, Kent K. Hathaway ${ }^{\mathrm{b}}$ \\ ${ }^{a}$ Engineering School of Sustainable Infrastructure \& Environment (ESSIE), 365 Weil Hall, University of Florida, \\ Gainesville, FL 32611. \\ ${ }^{b}$ US Army Corps of Engineers, Field Research Facility, Duck, North Carolina, USA.
}

\begin{abstract}
We investigate the performance of TRIADS, a numerical implementation of a phase-resolving, nonlinear, spectral model describing directional wave evolution in intermediate and shallow water. TRIADS simulations of shoaling waves generated by Hurricane Bill (2009) are compared to directional spectral estimates based on observations collected at the Field Research Facility of the US Army Corps Of Engineers, at Duck, NC. Both the ability of the model to capture the processes essential to the nonlinear wave evolution, and the efficiency of the numerical implementations are analyzed and discussed.
\end{abstract}

Keywords: TRIADS, directinal spectrum, nonlinear, Hurricane Bill.

\section{Introduction}

Nonlinear wave models developed for simulating wave propagation over large scales (100-1000 $\mathrm{km}$ ) in the deep ocean are based on a few fundamental wave-dynamics principles (deep-water paradigm): the propagation medium is isotropic and homogeneous; dispersion is strong; and wave nonlinearity is dominated by 4 -wave interactions. Relaxation processes (dispersion) dominate the nonlinearity allowing for an effective RPA closure (Random Phase Approximation, e.g., 3, 4, 39) of the nonlinear wave propagation problem. Four-wave interactions are the leading order nonlinear mechanism that contains resonances (e.g., Hasselmann 1962, 1963a, 1963b; 56, 57, 33). Although

\footnotetext{
${ }^{*}$ Corresponding author

Email addresses: alex.sheremet@essie.ufl.edu (Alex Sheremet), justin.r.davis@essie.ufl.edu (Justin R. Davis), mtian04.18@ufl . edu (Miao Tian), Jeffrey.L.Hanson@usace.army .mil (Jeffrey L. Hanson), mtian04.18@ufl.edu (Kent K. Hathaway)
} 
3-wave interactions (triads) are lower order and implicitly stronger, in deep water they are nonresonant, and thus irrelevant to large-scale dynamics. All wave models built around WAM's [54] central 4-wave interaction "engine" (the WAM-class models) follow the deep-water paradigm, whether they use the original numerical formulation the Direct Integration Approximation, DIA [22], or later updates, improvements, or alternatives (e.g., 55, 52, 53, 43, and many others). Examples of widely used WAM-class spectral models are SWAN Booij et al. [50], and WAVEWATCHIII, Tolman [51].

Nearshore wave transformation, however, belongs to a different paradigm. In the nearshore, nonisotropic/non-homogeneous bathymetric forcing induces a structural change of wave dynamics. As the water depth decreases, triads approach resonance and wave dispersion decreases. Increasingly efficient triads and weakening dispersion fundamentally change wave dynamics by allowing nonlinearity to build up, leading to the deformation of the wave shape, wave breaking, as well as to the generation of zero- and low-frequency oscillations (drift currents, wave set up, and infragravity (IG) waves [e.g., 12, 13, 26, 27, 28, 45, 46, 24, 29, 44, and many others]. In the frequency domain, this is expressed as energy transfers from the peak to higher and lower frequency bands, and the development of phase correlations between spectral bands, which invalidates the RPA description. Higher order statistics become important, for example, expressed by the skewness and asymmetry of the free surface elevation [e.g., 10, 30, 32]. Phase correlations are associated with the development of coherent structures such as cnoidal waves and trains of solitons [e.g., 37, 15, 16, 9, and many others], which undergo a complex and fascinating transformation eventually leading to wave peaking and breaking. The loss of isotropy and homogeneity invalidates standard wave-number Fourier representations. Nearshore-specific processes such as shoaling, reflection, refraction, and diffraction, also complicate the wave problem, transforming it into a boundary-value one, with significantly richer physics [e.g., 34].

In finite depth, (e.g., $(k h)^{2} \leq 0.5$, where $k$ is the characteristic wave number and $h$ is the local depth), both triad and quartet nonlinear mechanisms are relevant since quartets still control the dynamics of the high-frequency tail of the spectrum. Despite efforts to adapt the WAM-class of models to finite depth (e.g., SWAN), the lack of triad interactions ultimately renders them inadequate for nearshore applications(such as sediment transport) that require information about higher order statistics, or infragravity wave dynamics. The WAM-class models typically capture bulk sec- 
ond order statistics (such as total wave energy, and significant height) relatively well. As numerical modeling of wave propagation reaches maturity, and the demand for accurate wave forecasting in the nearshore increases, the availability of a numerical implementation of near-resonant triad interaction mechanism becomes paramount. While the formulation of phase-averaged shoaling models [e.g., 2, 25, 29, and others] is attractive for connecting naturally with deep-water models and addressing (at least in part) the lack of triad dynamics in the WAM-class models, the phase-averaging approach has inherent difficulties in capturing the nonlinear details of nearshore wave dynamics. The need to account for higher order statistics complicates closure formulations and forces the inclusion of evolution equations for bispectra (and possibly trispectra). The numerical resources needed for solving the nonlinear systen grow like powers of the number of modes (say $N$ ). Resolving the evolution of the directional spectrum alone (second order statistics) requires computing $O\left(N^{2}\right)$ nonlinear interaction terms. Resolving explicitly the bispectral evolution requires an additional system of $O\left(N^{2}\right)$ nonlinear equations. In shallow water, as the system approaches resonance in shallow water, the spectrum widens and the number of active modes increases [e.g., 8]; the validity of any closure approach degrades, and higher order closures are needed. Ultimately, the phase-averaged approach leads to a system of equations not much simpler than the original one, with the seemingly contradictory result of describing states that are strongly defined by phase information in terms phase-averaged quantities.

Phase-resolving spectral wave models e.g., 8, 11, 1, 2, 31, and others appear to achieve a certain balance between the brute-force approach of time-domain simulations (such as Boussinesq formulations, e.g., 49; or non-hydrostatic wave models 36,58 ) and the overly-elaborate approach of a phase averaged model incorporating high-order statistics. Their output is an explicit characterization of wave dynamics, that can be readily translated both into individual wave-field realizations, as well as into second and higher order statistics [11,2]. The effects of nonlinear interaction, dissipation, wave current interaction, etc, can also be examined directly and explicitly [e.g., 31, 47, 48, 32, and many others]. In contrast, the analysis of the time-domain model simulations requires the same kind of painstaking data analysis as field observations, with physical interpretation subject to similar uncertainties. Perhaps more importantly, phase-resolving models can handle situations where phase averaging is inconsistent, for example, in the case of narrow spectra, or when recurrence patterns occur. 
The nonlinear mild slope equation of Agnon and Sheremet [2] assumes a cylindrical (laterally uniform) beach mildly sloping in the cross shore direction. Let $x$ be the cross-shore, and $y$ the alongshore direction, with the vertical coordinate $z$ and the local depth $h(x)$. The free surface

Compared with their competitors, phase-resolving spectral models are also faster, which allows for covering larger spatial and temporal domains. This is essential for describing larger-scale infragravity waves generated through nonlinear interaction. Phase-resolving spectral models also connect in a natural way with the WAM-class deep-water models used extensively for deep-water wave forecasting.

This study investigates the performance of the phase-resolving, directional wave model TRIADS (Section 2) based on the nonlinear mild slope formulation proposed by Agnon and Sheremet [2]. It is not the goal of this study to educate the reader on the physics of near-resonant triad interactions (we refer the reader to the extensive literature on the subject, much of it cited above), or to promote the TRIADS model as an alternative to other existing models. We also do not present an exhaustive field testing of he numerical model presented. The model is in early stages of development, missing native formulations for important physics modules such as wave breaking, wind input/whitecapping, and others. We believe that in time, if the model will be used, these questions will be answered in a natural way. Instead, the goal of the work presented here is to demonstrate the application of TRIADS to a data set that exhibits a typical nonlinear shoaling transformation of a directional spectrum.

Details of its numerical implementation, as well as simulations of nonlinear shoaling of synthetic offshore over a plane beach are discussed by Davis et al. [7]. Here, field observations (Section 4) are used to validate the performance of the model, and study the impact of computational requirements (e.g., model resolution, run time, memory use) have on the numerical runs (Section 3). The performance of the model is presented in Section 5. Section 6 summarizes the results and discusses future possible directions of development. Details of some of the algebra and numerical implementation performance are given in the Appendix.

\section{Wave model}

\subsection{Governing equations}


elevation $\eta(x, y, t)$ is described as

$$
\eta(x, y, t)=\sum_{J} a_{J}(x, t) \exp i\left[\theta_{J}(x, t)+\kappa_{J} y-\omega_{J} t\right],
$$

94

where the index (mode)

$$
J=\left(\omega_{J}, \kappa_{J}\right)
$$

represents a discretization of the two-dimensional Fourier space $(\omega, \kappa) \in \mathbb{R}^{2}$, and $a_{J}$ and $\theta_{J}(x, t)$ are the modal amplitude and cross-shore phase, with $a_{-J}=a_{J}^{*}$, and $-J=(-\omega,-\kappa)$ (the asterisk denotes the complex conjugate). Under the assumption of the cylindrical beach, the frequency $\omega$ and the alongshore wave number $\kappa$ are invariants of propagation. At a given depth $h(x)$, the frequency and wave numbers satisfy the equations

$$
\omega^{2}=g K \tanh K h ; \quad K^{2}=k^{2}+\kappa^{2} ; \quad k=\frac{d \theta}{d x} .
$$

with $k$ the cross-shore component of the wave number vector $\mathbf{K}=(k, \kappa)$. The local direction of propagation of mode $J$ is uniquely determined by relations 3 . In equations $1-3$, the functions $h$, $k, K$, and $a$ are assumed to vary slowly with $x$ and $t$ (the mild-slope assumption). The directional evolution equation for the amplitudes of the Fourier modes is [e.g., 2, 7]

$$
\frac{d b_{J}}{d x}=-i \sum_{P, Q} W_{J,+P, Q} b_{P} b_{Q} e^{-i \Delta_{J, P, Q} \theta} \delta_{J, Q+P}+2 i \sum_{P, Q} W_{J,-P, Q} b_{-P} b_{Q} e^{-i \Delta_{J,-P, Q} \theta} \delta_{J, Q-P}
$$

where

$$
b_{J}=a_{J} c_{J}^{1 / 2} ; c_{J}=\left(\frac{k}{K} C\right)_{J} ; C_{J}=\left(\frac{\partial \omega}{\partial K}\right)_{J}, \text { and } \Delta_{J, \pm P, Q} \theta=\theta_{J} \mp \theta_{P}-\theta_{Q},
$$

with $c_{J}$ the cross-shore component of the modal group velocity $C_{J}, b_{-P}=b_{P}^{*}$ is the complex conjugate of $b_{P}$. In equation 4 the summation in the right-hand side is carried over all the modes in the discretization of the half-plane $(\omega, \kappa)$, with $\omega>0$. The triad selection criterion is represented by the Kronecker symbol

$$
\delta_{J, Q \pm P}= \begin{cases}1 & \text { if } J \mp P-Q=0 \\ 0 & \text { otherwise }\end{cases}
$$

with

$$
J \mp P-Q=0 \Leftrightarrow\left\{\begin{array}{l}
\omega_{J} \mp \omega_{P}-\omega_{Q}=0 \\
\kappa_{J} \mp \kappa_{P}-\kappa_{Q}=0
\end{array} .\right.
$$


The linear part of equation 4 represents the conservation of the linear cross-shore energy flux (note that $\left|b_{J}\right|^{2}=c_{J}\left|a_{J}\right|^{2}$ ). The interaction coefficient is

$$
W_{J, \pm P, Q}=\frac{1}{8} \frac{\sqrt{g} \sigma_{J}}{\sigma_{P} \sigma_{Q}}\left(c_{J} c_{P} c_{Q}\right)^{-1 / 2}\left( \pm 2 \mathbf{K}_{P} \cdot \mathbf{K}_{Q}+K_{P}^{2} \frac{\sigma_{Q}}{\sigma_{J}} \pm K_{Q}^{2} \frac{\sigma_{P}}{\sigma_{J}}+\sigma_{P}^{2} \sigma_{Q}^{2} \mp \sigma_{J}^{2} \sigma_{P} \sigma_{Q}\right)
$$

with $\sigma^{2}=\frac{\omega^{2}}{g}$, and $\mathbf{K}_{P} \cdot \mathbf{K}_{Q}=k_{P} k_{Q}+\kappa_{P} \kappa_{Q}=K_{P} K_{Q} \cos \alpha_{P Q}$, where $\alpha_{P Q}$ is the angle between the wave numbers of modes $P$ and $Q$.

Equations 3-8 are the core equations of the phase-resolving model TRIADS discussed in this study. They describe the stationary nonlinear transformation of a directional wave field propagating over a mildly-sloping, cylindrical beach. Laterally uniformity is implied within the accuracy of the governing equations, i.e., the alongshore variations should be of a higher order than the crossshore variations. Alongshore variations to the same order of magnitude as cross-shore ones can still be accommodated using a WKB-type expansions, but are not addressed here.

Because $K$ decreases with increasing depth (equation 3), choices of $(\omega, \kappa)$ are possible, that result for some $h(x)$ in

$$
k^{2}(\omega, \kappa, h)=K^{2}(\omega, h)-\kappa^{2} \leq 0 .
$$

These modes are trapped in the nearshore. The depth $h_{T p}$ for which $k^{2}(\omega, \kappa, h)=0$ is called the turning point. The model can only describe the evolution of the shoreward-propagating component of the trapped wave, and only in the spatial domain where the mode is a progressive wave. Therefore, modes that exhibit trapping (which have a turning point), are "active", i.e., participate in triad interactions in the domains where $h<h_{T p}$. All triads $(J, P, Q)$ that contain a trapped mode are ignored in the domain where $h>h_{T p}$. This is consistent with the negligible response of these modes to local forcing in the domain with $h \geq h_{T p}$ [e.g., 23].

The model accounts for linear processes such as refraction, shoaling, and for nonlinear triad interactions of shoreward propagating waves. Parametrizations of wave dissipation/growth processes can be readily incorporated. The obvious missing piece from this formulation is the 4-wave interaction. While it can be argued that near-resonant triads dominate the evolution over the short spatial scales of a typical beach (on the order of 10 wavelengths, e.g. 2), this is not true for the entire spectrum, because quartet dynamics are still expected to control the high-frequency modes, and thus should play an important role in the shoaling of coherent structures. In this respect, equa- 
tions 4 should be viewed as representing the current state of development, with quartet interaction to be included in the future.

\subsection{General properties of the governing equations}

Equations 3-8 are a generalization of the unidirectional model proposed by Freilich and Guza [8] to directional, dispersive wave fields [e.g., 1]. If dispersion is weak, equation 3 can be approximated as

$$
K=\left(\sigma h^{-1 / 2}\right)\left[1+\frac{1}{6} \sigma^{2} h+O\left(\sigma^{2} h\right)\right]
$$

and substituting the leading order term into equation 8 yields

$$
\lim _{h \rightarrow 0} W_{J, \pm P, Q}=\frac{\omega_{J}}{8 h}(g h)^{-3 / 4}\left[ \pm 2 \cos \alpha_{P Q} \pm 1\right]
$$

For $\alpha_{P Q}=0$ this is equivalent to the unidirectional resonant model of Freilich and Guza [8]. For the unidirectional case the following limits also hold,

$$
\lim _{h \rightarrow \infty} W_{J, P, Q}=0, \quad \text { and } \quad \lim _{h \rightarrow \infty} W_{J,-P, Q}=-\sqrt{2}\left(g \sigma_{J}^{3} \sigma_{P}^{3} \sigma_{Q}^{3}\right)^{1 / 2} \neq 0 .
$$

If $\alpha_{P Q} \neq 0$ the above relations are no longer valid.

The angle $\alpha_{P Q}$ affects the strength of the interaction also through the the cross-shore nonlinear oscillatory behavior of the governing equations. The efficiency of the nonlinear terms in equation 4 depends on the oscillations induced by the factor $\exp \left(i \Delta_{J, \pm P, Q} \theta\right)$, characterized by the detuning parameter [e.g., 14] (a function of the modes $P$ and $Q$, see Appendix)

$$
\mu_{ \pm P, Q}=\frac{\left|k_{J} \mp k_{P}-k_{Q}\right|}{K_{J}} .
$$

Assume that the triads in which mode $J$ participates can be divided into two groups: near-resonant triads, with $\mu=O(\varepsilon)$, and non-resonant ones with $\mu=O(1)$, where $\varepsilon$ is a small parameter, e.g., of the order of the terms neglected in the equation. Introducing explicitly slow and fast variables $X=$ $\varepsilon x$ and $\xi=x$, with characteristic length scales $\varepsilon^{-1} K_{J}^{-1}$ and $K_{J}^{-1}$, respectively, model amplitudes may be decomposed as [56]

$$
b_{J}=b_{J}^{(F)}(X)+\varepsilon b_{J}^{(B)}(x),
$$

where $b^{(B)}$ is the small, but fast-varying bound mode, and $b^{(F)}$ is the slow-varying one free mode. Because the right-hand side contains either near-resonant, or non-resonant terms, equation 4 can 
be separated into two pieces, one depending only on the slow variable $X$, and the other one only of the fast variable $\xi$, written formally as

$$
\begin{aligned}
\frac{d b_{J}^{(F)}}{d X} & =\sum_{P, Q}\left(-\delta_{J, Q+P}+2 \delta_{J, Q-P}\right) i W_{J, \pm P, Q} b_{ \pm P}^{(F)} b_{Q}^{(F)} e^{-i \Delta_{J, \pm P, Q} \theta} \\
& \mu=O(\varepsilon) \\
\frac{d b_{J}^{(B)}}{d \xi} & =\sum_{P, Q}\left(-\delta_{J, Q+P}+2 \delta_{J, Q-P}\right) i W_{J, \pm P, Q} b_{ \pm P}^{(F)} b_{Q}^{(F)} e^{-i \Delta_{J, \pm P, Q} \theta} \\
& \begin{array}{c}
\mu \\
=O(1)
\end{array}
\end{aligned}
$$

where the summation is carried over the triads that satisfy the conditions $\mu=O(1)$ and $\mu=O(\varepsilon)$ respectively.

The bound-wave equation is readily integrated to yield

$$
\begin{aligned}
b_{J}^{(B)} & =\sum_{P, Q}\left(\delta_{J, Q+P}-2 \delta_{J, Q-P}\right) \frac{W_{J, \pm P, Q}}{\Delta_{J, \pm P, Q} k} b_{ \pm P} b_{Q} e^{-i \Delta_{J, \pm P, Q} \theta} . \\
& \mu=O(1)
\end{aligned}
$$

Therefore, non-resonant interactions, $\mu=O(1)$ result in small bound-wave nonlinear corrections to free waves (equation 14), while resonant ones result in strong nonlinear exchanges across the wave spectrum.

In the shallow water limit $\mu_{ \pm P, Q}=0$ (resonant triad) if $(J, P, Q)$ are collinear, and the detuning varies weakly with the directional spread, with the effect that directional wave fields are dispersive even in a non-dispersive medium such as shallow water [40]. In finite depth frequency detuning effects dominate [e.g., 14] and all triads are non-resonant.

A corollary to this discussion is that if the wave field is strongly dispersive i.e. all triads satisfy $\mu=O(1)$, the model 4-8 behaves essentially like a linear model, since equation 15 contains no right-hand side. Alternatively, if the wave field is weakly dispersive, all modes are free waves and their interaction is completely described by equation 15 .

Because triads are resonant in the unidirectional, shallow-water approximation, the discussion of the characteristic properties of the governing equations in this case is relatively straightforward (e.g., the conservation of linear energy flux, Appendix). In non- or near-resonant conditions, due either to directional propagation $\left(\alpha_{P Q} \neq 0\right.$, e.g., 40) or to dispersive effects, flux conservation 
cannot be guaranteed through analytical approaches and to validate conservation one has to turn to numerical integration of the equations.

\section{Numerical implementation}

The current implementation of TRIADS is presented in Davis et al. [7]. For numerical integration purposes, the function $b_{J}$ is replaced by $B_{J}=b_{J} e^{i \theta_{J}}$ which yields the general equation

$$
\begin{gathered}
\frac{d B_{J}}{d x}=i\left(k_{J}+i \varepsilon_{J}\right) B_{J}-i \sum_{P, Q} W_{J, P, Q} B_{P} B_{Q} \delta_{J, P+Q}+2 i \sum_{P, Q} W_{J,-P, Q} B_{-P} B_{Q} \delta_{J,-P+Q},
\end{gathered}
$$

where the parameter $\varepsilon_{J}$ represents modal dissipation/growth rates, and can be used to incorporate parametrizations for breaking, wind forcing, bottom friction, and other processes. The version of the model run in this study includes only the depth-limited wave-breaking parametrization developed by Kaihatu and Kirby [30], with parameter values $B=1, \gamma=0.7$ and $F=0.5$ [47].

TRIADS is a FORTRAN 95/2003 code written to solve the the governing equations 3-8. The model (as of Version 1-50) contains approximately 40,000 lines of code, 47\% of which is the VODE ODE solver [5] and 12\% are testing routines. For the results presented here, the model has been compiled using the GNU FORTRAN compiler (4.9.2) and executed under the RHEL 6.6 Operating System (Red Hat Enterprise Linux; the model is functional under a variety of different computational environments; 7).

A run of the model is a Monte Carlo simulation consisting of a large number $N$ of numerical integrations of the initial value problem described in equations 3-8. Each integration is initialized with one "realization" of the Fourier coefficients $B_{J}$, constructed by assigning uniformly-distributed random phases (RPA approximation, e.g., 39) to modes $J$ whose amplitudes are defined by the directional spectrum given at the offshore boundary. Because individual realizations are independent, the numerical problem of generating a statistical ensemble is classified as an "embarrassingly parallel" problem [38]. In theory, such problems can reach speedup factors close to the number of cores or even faster if a run corresponding to a single RPA realization can fit within the cache of a processing core. However, in practice cores typically share a common system bus and thus, memory bandwidth can significantly limit performance.

Significant computational effort is spent in the calculation of the list of active triads $(J, P, Q)$. Because the triad list depends on the local depth, a direct numerical implementation of equation 
17 requires solving the triad-selection equations 6-7 at every call to the routine that computes the right-hand side of equation 4 . This accurate, but slow process can be sped up significantly (with a small loss of accuracy) if the model output grid is dense enough, such that the depth variation $\Delta h$ between two output points is much smaller that the local depth $h\left(\frac{\Delta h}{h} \ll 1\right)$. It can then be assumed that the triad list does not change significantly from one output point to the next. This idea is implemented as the "vectorized" engine of the model: for each integration between two output points, the list of triads is pre-computed based on the largest depth at the two output points. In its present implementation, the model includes two separate directional formulations, implementing these two approaches. The vectorized engine is 38 times faster than the direct implementation on a $(\omega, \kappa)$ grid of $60 \times 133$ modes. An additional, separate formulation is included for unidirectional propagation, to take advantage of the considerable memory and numerical effort saved in this case. Over a grid of 60 frequency modes, the unidirectional formulation is 193 times faster than a simulation performed using 60 frequencies and 133 alongshore wave numbers.

The detuning effect (Subsection 2.2) can be used as a tool to correct the tendency of the governing equations 3-8 to overestimate the scope of the nonlinear coupling. Because equation 17 is not "scale-aware", it includes a large number of triads that are far from resonance, $\mu=O(1)$, and thus either irrelevant or physically meaningless (e.g., very high frequency waves forcing IG waves). A straightforward numerical implementation of the model 3-8 will attempt to compute the interaction every possible triad, regardless of the detuning state, resulting in a significant waste of resources and contaminating the integration with numerical-truncation noise. Triads that are far from resonance can be excluded from integration by setting a cutoff detuning value $\mu_{C r}$, such that only triads with $\mu<\mu_{C r}$ are integrated. Note that, if an "optimal" value of $\mu_{C r}$ exists, it must be application-specific. For example, we expect optimal $\mu_{C r}$ to depend on the size of the integration domain, the beach profile and the spectral distribution. For the simulations shown below, the model performance was tested for several values (Figure 1). Using large $\mu_{C r}$ values accounts for triads increasingly far from resonance and has the consequence of increasing simulation time. The value chosen for all the numerical simulations presented here, $\mu_{C r}=5$, included all but the most non-resonant triads. Further details on the numerical performance of the model are given in the Appendix. 


\section{Field Observations}

Testing TRIADS is currently a challenge, due to the scarcity of observations of directional spectral evolution in the nearshore, and in particular the lack of phase-resolving observations. In this study, the model was tested using field observations collected during Hurricane Bill in August 2009, at Duck NC.

The US Army Corps of Engineers Field Research Facility (FRF) maintains an operational crossshelf array of meteorological, directional wave and current sensors for continuous observation of wave and current conditions in the energetic shelf environment off Duck, North Carolina [17, 18]. The array consists of 4 bottom-mounted NORTEK Acoustic Wave and Current (AWAC) profilers at depths of 5, 6.5, 8.5 and $11.2 \mathrm{~m}$ (Figure 2a), and two Datawell Directional Waverider buoys at 17- and 26-m depths. An instrument tower at the end of the FRF pier (8-m water depth) includes a meteorological station with an anemometer (RM Young Model 09101) located at an elevation of $19.5 \mathrm{~m}$. The wave stations are in a direct line with a 3-m discus buoy (National Data Buoy Center station 44014) that includes a meteorological station and directional wave sensor at 48-m depth. In its entirety, the array spans $95-\mathrm{km}$ and captures all phases of wave transformation from the outer continental shelf to within the surf zone.

The cross-shore AWAC array collect 34 minutes records hourly with a $2 \mathrm{~Hz}$ sampling rate. The FRF developed custom spectral processing routines [18] for these gauges that uses a combination pressure, vertical acoustic surface track beam (AST), three oblique ( $25^{\circ}$ off vertical) beam velocities, and computed orthogonal orbital current velocities. The AST signal samples at $4 \mathrm{~Hz}$, twice that of the oblique beams, and provides measurements of surface displacement. Spectral analysis at lower frequency end of the spectrum uses IMLE processing of the orbital currents and the AST, which is treated as a point measurement. The upper end of the spectrum uses IMLE processing with the AST and individual radial-velocity beams as a spatial array. The mid portion of the spectrum uses a linear merge of the two spectra. This spectral reconstruction technique was shown to reduce directional spreading at the high and low ends of the spectra. If the AST signal fails quality checks the pressure signal is substituted. The AST will lose track of the surface when wave breaking (dissipation) injects air bubbles into the water column. Wave data are collected hourly in 34-minute records at 2- $\mathrm{Hz}$ sampling rate. 
The FRF also has a phased array (8m Array) of pressure sensors with dimensions of 120m crossshore and $255 \mathrm{~m}$ alongshore at $8 \mathrm{~m}$ depth. The array design was based on the work of Davis and Regier [6] and uses the Iterative Maximum Likelihood Method (IMLM) for the wave analysis [42, 41, 35]. Data are collected in 136 minute records ( $2 \mathrm{hr}, 16 \mathrm{~min})$ every three hours, sampled at $2 \mathrm{~Hz}$. Since this array has a relatively large number of elements and spatially fixed, not requiring a magnetic compass for orientation, the directional wave spectra appear more realistic than those provided by the AWAC gauges.

Since becoming operational in 2007, the FRF cross-shelf array has observed a wide variety of high-energy wave conditions, including Category 3 hurricanes (Hurricanes Irene and Sandy) and multiple Nor'easters. The analysis presented here focuses on the nearshore waves generated by Hurricane Bill. Hurricane Bill evolved quickly from a tropical-wave state in the eastern Atlantic (August 15, 2009) to Tropical Storm and became a Category 1 hurricane on August 17, 2009. The center of the storm passed the Duck shelf area approximately $650 \mathrm{~km}$ offshore with sustained Category 2 winds, generating a low frequency swell (peak period 16 s, Figure 2b). During the period from August 21st 17:00 hr to August 22 18:00 hr, with wave heights $<2 \mathrm{~m}$ at AWAC 1 (Figure 2b), the cross-shore fluxes estimated using the AWAC records are approximately equal (Figure 2c), indicating that the array is in the shoaling zone. At the peak of the storm (wave heights $>2 \mathrm{~m}$ at AWAC 1), the strong flux dissipation suggests that the AWAC array was in the surf zone.

Because the main goal of this study is to test the performance of the nonlinear triad interaction mechanism (essential for nonlinear shoaling processes), and to minimize the influence of parametrizations of various physical processes either not yet tested with this model (e.g., depthlimited breaking), or not implemented (e.g., wind input, white-capping, and bed friction), numerical simulations are limited to the region covered by the AWAC array, spanning 11-m to 5-m depth, and to observations on the shoaling regime.

\section{Model results and discussion}

We illustrate the performance of the model using simulations for the observations collected on August 22nd, 02:00 hr, with the AWAC array in the shoaling zone (red circle in Figure 2c). Other test runs of TRIADS in this shoaling regime (August 21st 17:00 hr to August 22nd 18:00 hr) preformed similarly. 
The directional spectrum estimated at AWAC 1 was resampled and interpolated (see the discussion in 7) into a discretization of 60 frequencies by 133 alongshore wave numbers (a total of 7980 modes; see also the Appendix). The results presented here represent averages over $N=100$ realizations of shoaling transformation of the directional spectrum estimated at AWAC 1. Because the initial directional spectrum contains no information about phase correlations in the IG frequency band, IG modes were initialized with bound waves (equation 16). Each realization was integrated from the offshore up to $2 \mathrm{~m}$ depth. Model output points were distributed arbitrarily (Figure 2), but in a denser pattern in shallow water, to better resolve the increased triad activity in shallow water. At each output point the model provided modal amplitudes, dissipation rates, nonlinear transfer rates, etc.

The main feature of the nonlinear shoaling transformation of the directional spectrum is the generation of the second and higher harmonics of the peak, and the weak nonlinear amplification of the IG band due to energy transfers from swell frequencies. TRIADS captures well the nonlinear trend but the simulated peak harmonic has a narrower directional-spread, and the domain of the higher harmonics is smoother than the observed spectrum (Figures 3-6), an effect of limited number of frequency modes used.

The directional spread of the AWAC spectral estimates, however, appears to be uniformly wide regardless of the location, which suggests that the narrower directional distribution of the simulated spectrum is more realistic. Indeed, independent observations at the FRF 8-m array (Figure 5a, compare with Figure 3c) have a half-width of approximately $30 \mathrm{deg}$. based on a $10^{-2}$ decay from the spectral peak, whereas the AWAC half width based on the same measure is $180 \mathrm{deg}$. Perhaps more importantly, the 8-m array does not exhibit leakage into the trapped-wave directions. (Note the slow generation in the numerical simulations of trapped IG models propagating at wide angles (Figure 4d).)

For the test presented here, initializing the model at the AWAC 1 location (11.3-m depth) has the advantage of minimizing the contribution of untested physics modules. However, for a wave of 16 s, 11.3-m depth is rather shallow. The linear theory yields a "local" wave length of approximately $170 \mathrm{~m}$, with a dispersion parameter $(K h)^{2}=0.18$, and an nonlinearity parameter of approximately $\frac{a}{h}=0.09$. At AWAC 1 the wave field is already significantly nonlinear, which implies that phase correlations already exist, and the RPA hypothesis is to a certain degree invalid. This is confirmed 
by the peak harmonic already present at the AWAC 1 location and that continues to develop in shallower water (Figures 3-4). The initialization of the IG band with bound waves represents only a partial correction, since the phase correlations likely span the entire spectrum. Despite the relative weak nonlinear aspect of the spectral shapes, the nonlinear character of wave transformation is remarkably evident in the deformation of the free surface elevation shape, especially in the development of wave skewness (Figure 7).

One of the effects of the violation of the RPA initialization constraint is apparent in the behavior of bulk cross-shore energy fluxes (Figures 8-9). For a linear evolution and in the absence of dissipation/growth sources, the cross-shore energy flux is conserved. The total flux remains approximately constant (model conserves the total cross-shore flux) but for the last approximately $100 \mathrm{~m}$ of the integration domain, where breaking dissipation becomes active (see also Figure 11). The simulated evolution of IG $\left(f<0.5 f_{\text {Peak }}\right)$, swell $\left(0.5 f_{\text {Peak }} \leq f<1.5 f_{\text {Peak }}\right)$ and seas $\left(f \geq 1.5 f_{\text {Peak }}\right)$ is strictly due to nonlinear interactions, showing the expected overall trend, with the swell band losing energy to the seas and IG bands (see also Figure 9a-b for spectral details). Note that simulated seas band overshoots the growth indicated by observations. This is the expression of an initial internal phase "adjustment" in the model to the initial violation of the RPA hypothesis, through which phase correlations are generated and the seas band transitions some of the initial free-mode flux to a bound-mode state. This trend is evident also in the behavior of the local nonlinear transfer rates (Figure 10), calculated based on equation 17 as

$$
D_{J}^{+}=\mathfrak{I}\left\{\sum_{P, Q<J} \frac{W_{J, P, Q} B_{P} B_{Q}}{B_{J}} \delta_{J, P+Q}\right\}, \quad D_{J}^{-}=-\mathfrak{I}\left\{\sum_{J<P, Q} \frac{2 W_{J,-P, Q} B_{-P} B_{Q}}{B_{J}} \delta_{J,-P+Q}\right\},
$$

where $D_{J}^{+}$represents the rate of energy-flux transfer to mode $J$ from the left (from lower frequencies), $D_{J}^{-}$represents the rate of energy flux transfers to mode $J$ from the right (from higher frequencies), and $\mathfrak{I}\{z\}$ is the imaginary part of the complex number $z$. The transfer rates illustrate the process of the generation of peak harmonics and IG wave, with $D_{J}^{+}>0$ in the seas band, and $D_{J}^{-}>0$ in the IG band. The initial adjustment of the model is visible in the strong initial growth and subsequent slow oscillation of the $D_{J}^{+}$rates in the frequency band corresponding to the peak harmonic. The bulk transfer rates (integrated over frequency bands; Figure 10fg) show that while exchanges occur recursively across the spectrum over the entire integration domain, significant net 
transfers from the swell to seas and swell to IG occur in water depth less than $10 \mathrm{~m}$, in front of the bar. The effect of the weak unshoaling shoreward of the bar is also apparent in the weakening or even change of sign of the bulk transfer rates.

\section{Summary}

This study discusses the first test performed with field observations of TRIADS, a phase-resolving model for nonlinear shoaling of a directional wave spectrum on a cylindrical beach. Field observations were provided by a unique cross-shelf wave transformation array deployed by the USACE at the Field Research Facility at Duck, NC. Because the focus of this first test was evaluating the performance of the model nonlinear interaction "engine", the data set of observations used for comparison were selected to reduce the impact of other physical processes. The effect of wind input, white-capping, and bottom friction were minimized by restricting the simulations to the area covered by the nearshore (AWAC) part of the FRF observation array.

The performance of the model is illustrated for a strongly nonlinear shoaling event recorded during Hurricane Bill in August 2009. The spatial and temporal coverage and resolution of the unique data set provided by FRF allowed us to select a time window in which the nearshore AWAC array was in the shoaling region, with the wave field a rather clean directional swell, thus minimizing the depth-limited breaking effects. The test run highlighted the limitations of the data-model comparison effort, mainly stemming from spatial and temporal resolution constraints, such as model initialization at a location too shallow for the type of waves studied, overestimated directional spread in the observations.

Despite limitations and constraints, the simulations compare well with the observations both in directional character and frequency distribution, capturing the overall shape of the spectrum, and also describing more subtle nonlinear trends such as the growth of the IG band cross-shore energy flux, the broadening of the frequency spectrum, and the generation of peak harmonics.

Wave conditions during Hurricane Bill are well suited for the applications of TRIADS to the FRF AWAC observation array, with shallowest sensor (near the 5-m isobath) typically too deep for normal storms. Demonstrating the model capabilities using these data does not imply that the model application is limited to hurricane conditions. As a precursor of depth-limited breaking, 
the nonlinear shoaling process always occurrs immediately seward of the surf zone, in the socalled shoaling zone. TRIADS is derived from directly from the nonlinear governing equations and as such it is applicable to the shoaling zone wherever the limiting assumptions of cross-shore milde slope and alongshore uniformity on the characteristic wave scale apply. The intensity of the nonlinear process can vary depending on the beach slope and other dissipation processes such as bottom friction, or wind input and whitecapping. We stress that while triad dynamics strengthens in general as the spectrum shoals, shoaling is a non-local process, in which the accumulation of phase correlations during propagation is important.

Future work will focus on alleviating known limitations of the model (lack of physics modules, lack of nonlinear quartets, lack of trapped-wave dynamics, etc), and seeking additional testing opportunities.

\section{Appendix}

\subsection{Detuning}

In the shallow water limit 10 the selection criteria 7 acquire the simple form

$$
\left\{\begin{array}{l}
K_{J} \mp K_{P}-K_{Q}=0 \\
\kappa_{J} \mp \kappa_{P}-\kappa_{Q}=0
\end{array},\right.
$$

allowing for an explicit, albeit tedious, investigation of some of the properties of the governing equations 3-8.

Assuming that the modes participating in a given triad are leaky, i.e., $|\kappa| \leq K=\frac{\sigma}{\sqrt{h}}$ for each of $J, P$, and $Q$, the detuning coefficient is a function of four independent parameters (modes $P$ and $Q$ )

$$
\mu_{ \pm P, Q}=\frac{1}{K_{Q} \pm K_{P}}\left|\sqrt{\left(K_{Q} \pm K_{P}\right)^{2}-\left(\kappa_{Q} \pm \kappa_{P}\right)^{2}}-\left(K_{Q} \cos \alpha_{Q} \pm K_{P} \cos \alpha_{P}\right)\right|,
$$

where $\alpha_{P, Q}$ are the angles of propagation with respect to the shore normal direction. Because the governing equations describe only modes that that propagate shoreward, we assume in the sequel that $0 \leq \alpha_{P, Q}<\frac{\pi}{2}$.

From equation 20, it is obvious that $\mu_{ \pm P, Q}=0$ for a collinear triad, i.e., for $\alpha_{P}=\alpha_{Q}$. Due to the number of independent parameter, a complete discussion of the detuning is complicated even in 
the shallow-water case. However, some simple properties can be readily obtained if the some of the parameters are fixed. For example, setting the direction of mode $Q$ to be shore-normal, i.e., $\alpha_{Q}=0$ and $\kappa_{Q}=0$, equation 20 becomes

$$
\mu_{ \pm P, Q}=\frac{1}{K_{Q} \pm K_{P}}\left|\sqrt{\left(K_{Q} \pm K_{P}\right)^{2}-\kappa_{P}^{2}}-\left(K_{Q} \pm K_{P} \cos \alpha_{P}\right)\right|,
$$

where the angle $\alpha_{P}$ is the angle between the direction of propagation of modes $P$ and $Q$. The detuning for a sum interaction $J=P+Q$ with $\alpha_{P} \simeq \frac{\pi}{2}, \kappa_{P} \simeq K_{P}$, yields

$$
\mu_{P, Q} \simeq \frac{1}{K_{Q}+K_{P}}\left|\sqrt{\left(K_{Q}+K_{P}\right)^{2}-K_{P}^{2}}-K_{Q}\right|=\frac{K_{Q}}{K_{J}}\left|\sqrt{1+2 \frac{K_{P}}{K_{Q}}}-1\right|
$$

For the case of second-harmonic generation case, setting $K_{J}=2 K_{Q}=2 K_{P}$ yields $\mu=0.366$. This suggests that detuning depends only weakly on the directional spread. The dependency is illustrated in Figure12. For difference interactions a more careful analysis is required, because mode $J$ can become trapped (the quantity under the square root becomes negative). The effect of the directionality on the efficiency of nonlinear triad shoaling is illustrated in Figures 13-14.

\subsection{Energy flux conservation}

In shallow water, the equation 4 conserves the total linear energy flux for shore-normal propagation. To show this, revert to free surface elevation amplitudes, and write the equation 4 as

$$
C_{J} \frac{d a_{J}}{d x}+\frac{1}{2} \frac{d C_{J}}{d x} a_{J}=-i \sum_{P, Q} V_{J, P, Q} a_{P} a_{Q} e^{-i \Delta_{J, P, Q} \theta} \delta_{J, Q+P}
$$

where a mode $J=\left(\omega_{J}, \kappa_{J}=0\right)$ is now represented only by its radian frequency $\omega_{J} \in \mathbb{R}$, and the interaction coefficient is

$$
V_{J, P, Q}=\frac{1}{4} \frac{\sqrt{g} \sigma_{J}}{\sigma_{P} \sigma_{Q}}\left(2 K_{P} K_{Q}+K_{P}^{2} \frac{\sigma_{Q}}{\sigma_{J}}+K_{Q}^{2} \frac{\sigma_{P}}{\sigma_{J}}+\sigma_{P}^{2} \sigma_{Q}^{2}-\sigma_{J}^{2} \sigma_{P} \sigma_{Q}\right) .
$$

Multiplying equation 23 by $a_{J}^{*}$ and adding the result to its complex conjugate, and summing over all modes yields

$$
\frac{d}{d x}\left(\sum_{J} \frac{1}{2} C_{J}\left|a_{J}\right|^{2}\right)=-2 \mathfrak{I}\left\{\sum_{J, P, Q} V_{J, P, Q} a_{J} a_{P}^{*} a_{Q}^{*} e^{i \Delta_{J, P} \theta} \delta_{J, Q+P}\right\}
$$


To include all three variants of each triad, i.e. $(J, P, Q) \delta_{J, P+Q},(P,-Q, J) \delta_{P,-Q+J}$, and $(Q,-P, J) \delta_{Q,-P+J}$, the equation 25 can be rewritten as

$\frac{d}{d x}\left(\sum_{J} \frac{1}{2} C_{J}\left|a_{J}\right|^{2}\right)=-\frac{2}{3} \mathfrak{I}\left\{\sum_{J, P, Q}\left(V_{J, P, Q}+V_{-P, Q,-J}+V_{-Q, P,-J}\right) a_{J} a_{P}^{*} a_{Q}^{*} e^{i \Delta_{J, P, Q} \theta} \delta_{J, P+Q}\right\}=0$,

because $\left(V_{J, P, Q}+V_{-P, Q,-J}+V_{-Q, P,-J}\right) \delta_{J, P+Q}=0$, as can be verified through straightforward algebra.

\subsection{Numerical performance of the current implementation of TRIADS}

The model performance was assessed using three sizes of the $(\omega, \kappa)$ domain: 1$)$ low, $25 \times 51$ modes; 2) medium, $40 \times 93$ modes; and 3) large $60 \times 133$, where the first number represents the number of frequencies and the second the number of alongshore wave numbers. The three sizes also correspond to low, medium, and high resolutions, as the limits of the maximum values of the frequency and wave numbers were kept constant. The comparisons of simulations and observations presented in Section 6 are based on high-resolution runs. Low- and medium-resolution grids were used for testing various components of the model.

Two computational platforms based on different types of hardware were used for testing (Table 1). The 4-core Dell Optiplex has a higher clock rate $(3.5 \mathrm{GHz})$; with 24-core, the 2.7-GHz Dell Precision is the more expensive platform. To minimize the effect of non-computational factors (e.g., disk speed), the recorded execution times reflect the amount of time spent for integrating the ODE system (97\% of the total execution time).

Computationally dense segments of the code were wrapped in OpenMP parallelization constructs enabled via CPP directives. In particular, several sets of constructs were added to the routine which computes the right hand side equation 17. Tests were performed to assess the impact of these directives on by comparing the execution time of the model compiled with the constructs disabled (serial version of the model) with the model compiled with the constructs enabled (the OpenMP parallel version of the model), but using only one processor. For the three domains tested, these serial vs. parallel times were within $1 \%$ of each other. All execution times reported (for 1, 2, 4, etc. processors) here were made with the OpenMP constructs enabled. 
In single-core/single-realization runs (Table 2), the OpenMP parallel version of TRIADS was 37\% faster on the Dell Optiplex than the Dell Precision for a single core simulation. The Precision was, however, approximately 2.5 times faster for the medium and high resolution domains when multiple-cores were used. Although in absolute terms a larger number of cores reduces the run time of an individual realization, this approach to speeding up computations is not efficient. Here, the efficiency is defined as the speed increase (normalized by the number of cores for a single run)

$$
e_{1}\left(n_{c}\right)=\frac{1}{n_{c}} \frac{T_{S}(1)}{T_{s}\left(n_{c}\right)} ; \quad e_{2}\left(n_{i}\right)=\frac{T_{s}(1)}{T_{s}\left(n_{i}\right)}
$$

where $e_{1}$ and $e_{2}$ are the efficiency for single and multiple runs; $T_{s}\left(n_{c}\right)$ is the solver run time for $n_{c}$ cores; and $T_{S}\left(n_{i}\right)$ is the solver run time for $n_{i}$ realizations. For example, the high resolution domain, which contains the most amount of computational work, only has a $44.3 \%$ efficiency when using 24 cores.

In a single-core/multiple-realization runs (Table 3), the efficiency of platforms improved significantly compared to the OpenMP version of TRIADS. For high resolution simulation the efficiency increased from 65.2 .5 to $79.8 \%$ on the 4-core Optiplex, and from 44.3 to $97.7 \%$ on the 24 -core Precision. The $121 \%$ improvement on the Precision was likely due to the Xeon architecture which supports very high memory bandwidths (at a price).

Comparing the performance of the two platforms, and their cost effectiveness (Table 4) shows that simulation time scales well with the number of triads, with the time per triad being approximately the same on both platforms, and independent of domain discretization. Per processing core, the Dell Optiplex was 37\% faster, as well as 58-60\% cheaper.

The maximum resident set size (the maximum amount of physical memory used by the model) was 41.0, 367.2 and 1425.2 MB for the $20 \times 51,40 \times 93$, and $60 \times 133$ domains (normalized values: $1,8.9$, and 34.7). These values indicate that amount of memory used scales by a factor in between the number of modes and the number of triads (consistent with the normalized number of modes and triads, Table 4).

Acknowledgments. This research was supported by ONR Grants N00014-13-1-0620, N00014-101-0389, and N00014-10-0805. 


\section{References}

[1] Agnon, Y., A. Sheremet, J. Gonsalves, and M. Stiassnie (1993), A unidirectional model for shoaling gravity waves, Coastal Eng. 20, 29-58.

[2] Agnon, Y., and A. Sheremet (1997), Stochastic nonlinear shoaling of directional spectra, J. Fluid Mech. 345, 79-99.

[3] Benney, D.J., and P.G. Saffman (1966), Nonlinear interactions of random waves. Proc. R. Soc. Lond. A 289, 301-321.

[4] Benney, D. J. and A.C. Newell (1969), Random waves closures. Stud. Appl. Maths 1, 32-57.

[5] Brown, P.N., G. D. Byrne, and A. C. Hindmarsh (1989), VODE: a variable-coefficient ODE solver. SIAM J. Sci. Stat. Comput. 10, 5 (September 1989), 1038-1051. DOI=10.1137/0910062 http://dx.doi.org/10.1137/0910062

[6] Davis, R.E. and L, Regier, 1977. Methods of estimating directional spectra from multi-element arrays. J. Mar. Res., 35, 453.

[7] Davis, J.R., A. Sheremet, M. Tian, and S. Saxena (2014), A Numerical Implementation of a Nonlinear Mild Slope Model for Shoaling Directional Waves, J. Mar. Sci. Eng. 2, 140-158; doi:10.3390/jmse2010140

[8] Freilich M.H., and R.T. Guza (1983), Nonlinear effects of shoaling surface gravity waves Phil. Trans. R. Soc. Lond. A 311, 1-41.

[9] El, G.A., R.H.J. Grimshaw, and W. K. Tiong (2012), Transformation of a shoaling undular bore, J. Fluid Mech.709, 371-395 doi:10.1017/jfm.2012.338.

[10] Elgar, S., Guza, R.T., (1985), Observations of bispectral of shoaling surface gravity waves. J. Fluid Mech. 161, 425-448.

[11] Elgar, S., and R.T. Guza (1986), Nonlinear model predictions of bispectra of shoaling surface gravity waves, J. Fluid Mech 167, 1-18. 
[12] Elgar, S., T.H.C. Herbers, M. Okihiro, J. Oltman-Shay, and R.T. Guza (1992). Observations of infragravity waves. J. Geophys. Res. 97, 15573-15577.

[13] Elgar, S., T.H.C. Herbers, R.T. Guza (1994), Reflection of ocean surface gravity-waves from a natural beach. J. Phys. Oceanogr. 24 (7), 1503-1511.

[14] Elgar, S., R.T Guza, and M.H. Freilich (1993), Observations of Nonlinear Interactions in Directionally Spread Shoaling Surface Gravity Waves, J. Geophys. Res., 98 (Cll), 20,299-20,305.

[15] Grimshaw, R. (1970), The solitary wave in water of variable depth. Journal of Fluid Mechanics, 42, pp 639-656 doi:10.1017/S0022112070001520

[16] Grimshaw, R. (1971), The solitary wave in water of variable depth. Part 2. Journal of Fluid Mechanics, 46, pp 611-622 doi:10.1017/S0022112071000739

[17] Hanson, J.L., H. C. Friebel, and K. K. Hathaway (2010), Coastal Wave Transformation: Observations and Modeling, WISE 2010, 17th Waves in Shallow water Environment Meeting, Brest, France.

[18] Hathaway, K. K, and J. L. Hanson (2011), Directional wave spectra analysis from a crossshore array of acoustic Doppler profilers, 12th International Workshop on Wave Hindcasting and Forecasting, Hilo, Hawaii.

[19] Hasselmann, K. (1962), On the non-linear energy transfer in a gravity-wave spectrum. 1. General theory, J. Fluid Mech. 12/4, 481-500.

[20] Hasselmann, K. (1963), On the non-linear energy transfer in a gravity wave spectrum. 2. Conservation theorems - wave-particle analogy - irreversibility, J. Fluid Mech. 15/2, 273-281.

[21] Hasselmann, K. (1963), On the non-linear energy transfer in a gravity-wave spectrum. 3. Evaluation of the energy flux and swell-sea interaction for a Neumann spectrum, J. Fluid Mech. 15/3, 385-398.

[22] Hasselmann, S., K. Hasselmann, J.H. Allender, and T.P. Barnett (1985), Computations and parametrizations of the nonlinear energy transfer in a gravity-wave spectrum. Part 2. 
Parametrizations of the nonlinear energy transfer for application in wave models, J. Phys. Oceanogr. 15, 1378-1391.

[23] Henderson, S.M., and A.J. Bowen (2002), Simulations of Dissipative, Shore-Oblique Infragravity Waves, J. Phys. Oceanog. 33(8), 1722-1732, doi: http://dx.doi.org/10.1175/2398.1.

[24] Henderson, S.M., R.T. Guza, S. Elgar, T.H.C. Herbers, A.J. Bowen (2006), Nonlinear generation and loss of infragravity wave energy. J. Geophys. Res. 111 (C12) Article C12007.

[25] Herbers, T.H.C., Burton, M.C. (1997), Nonlinear shoaling of directionally spread waves on a beach. J. Geophys. Res. 102 (C9), 21101-21114.

[26] Herbers, T.H.C., S. Elgar, R.T. Guza (1994), Infragravity-frequency (0.005-0.05 Hz) motions on the shelf. 2. Free waves. J. Phys. Oceanogr. 24 (5), 1063-1079.

[27] Herbers, T.H.C., S. Elgar, R.T. Guza, W.C. O'Reilly (1995a). Infragravity-frequency (0.005-0.05 Hz) motions on the shelf. 2. Free waves. J. Phys. Oceanogr. 25 (6), 917-927.

[28] Herbers, T.H.C., S. Elgar, R.T. Guza (1995b), Generation and propagation of infragravity waves. J. Geophys. Res. 100 (C12), 24863-24872.

[29] Janssen, T.T., Herbers, H.C., Battjes, J.A. (2006), Generalized evolution equations for nonlinear surface gravity waves over two-dimensional topography. J. Fluid Mech. 552, 393-418.

[30] Kaihatu, J.M., and J.T. Kirby (1995), Nonlinear transformation of waves in finite water depth, Phys. Fluids 7, 1903-1914.

[31] Kaihatu, J.M. (2009), Application of a nonlinear frequency domain wave-current interaction model to shallow water recurrence effects in random waves, Ocean Modelling 26, 190-205.

[32] Kaihatu, J.M, and N. Tahvildari (2012), The combined effect of wave-current interaction and mud-induced damping on nonlinear wave evolution, Ocean Modelling, Volume 41, 2012, Pages 22-34, ISSN 1463-5003, http://dx.doi.org/10.1016/j.ocemod.2011.10.004.

[33] Krasitskii, V.P. (1994), On the reduced equations in the Hamiltonian theory of weakly nonlinear surface waves, J. Fluid Mech. 272, 1-20. 
[34] LeBlond, P.H., and L.A. Mysak (1978), Waves in the ocean, Elsevier Oceanography Series 20, Elsevier Scientific Publishing Co.

[35] Long, C. E., and J.M. Oltman-Shay, 1993. Preliminary estimates of frequency-direction spectra derived from the SAMSON pressure gage array November 1990 to May 1991, USACE Tech Report CERC-93-1.

[36] Ma, G., F. Shi, and J.T. Kirby (2012), Shock-capturing non-hydrostatic model for fully dispersive surface wave processes, Ocean Modelling 43-44, 22-35.

[37] Madsen, O. S. and C. C. Mei, 1969, The transformation of a solitary wave over an uneven bottom, J. Fluid Mech. 39 , 781-791.

[38] Moler, C. (1986), Matrix Computation on Distributed Memory Multiprocessors. Hypercube Multiprocessors (Society for Industrial and Applied Mathematics, Philadelphia) Heath, Michael T., ed. ISBN 0898712092.

[39] Nazarenko, S. (2011), Wave Turbulence, Lecture Notes in Physics 825, Springer-Verlag.

[40] Newell, A.C., and P.J. Aucoin (1971), Semidispersive wave systems. J. Fluid Mech. 49, 593-609.

[41] Oltman-Shay J., R.T. Guza (1984), A data-adaptative ocean wave directional-spectrum estimator for pitch-roll type measurements. J. Phys. Oceanogr., 14, 1800-1810.

[42] Pawka, S.S. (1983), Island shadows in wave directional spectra, J. Geophys. Res., 88, C4, 2579-259.

[43] Resio, D.T., and W. Perrie (2008), A two-scale approximation for efficient representation of nonlinear energy transfers in a wind wave spectrum,. Part 1: Theoretical development, J. Phys. Oceanogr. 38/12, 2801-2816.

[44] Ruessink, B.G., 1998. The temporal and spatial variability of infragravity energy in a barred nearshore zone. Cont. Shelf Res. 18 (6), 585-605. 
[45] Sheremet, A., R.T. Guza, S. Elgar, and T.H.C. Herbers (2002), Observations of nearshore infragravity waves: seaward and shoreward propagating components. J. Geophys. Res. 107 (C8), 3095. doi:10.1029/2001JC000970.

[46] Sheremet, A., R.T. Guza, and T.H.C. Herbers (2005), A new estimator for directional properties of nearshore waves. J. Geophys. Res. 110 (C1) Article: C01001.

[47] Sheremet, A., J.M. Kaihatu, S.-F. Su, E.R. Smith, and J.M. Smith (2011), Modeling of Nonlinear Wave Propagation Over Fringing Reefs, Coastal Eng. 58, 1125-1137.

[48] Sheremet, A., S. Jaramillo, S.-F. Su, M. A. Allison, and K. T. Holland (2011), Wave-mud interaction over the muddy Atchafalaya subaqueous clinoform, Louisiana, United States: Wave processes, J. Geophys. Res., 116, C06005, doi:10.1029/2010JC006644.

[49] Shi F., J.T. Kirby, J.C. Harris, J.D. Geiman and S.T. Grilli (2012), A high-order adaptive time-stepping TVD solver for Boussinesq modeling of breaking waves and coastal inundation, Ocean Modelling, 43-44, 36-51.

[50] Booij, N., R.C. Ris, and L.H. Holthuijsen (1999), A third-generation wave model for coastal regions: 1. Model description and validation. J. Geophys. Res. 1999, 104, 7649-7666.

[51] Tolman, H. L. (1991), A third generation model for wind on slowly varying, unsteady and inhomogeneous depth and currents, J. Phys. Oceanogr., 21, 766-781.

[52] Tracy, B.A., and D.T. Resio (1982), Theory and calculation of the nonlinear energy transfer between sea waves in deep water, U.S.Army Engineer Waterways Experiment Station WES, Rep. 11, 52 pp, 1982.

[53] Van Vledder, G.Ph. (2006), The WRT method for the computation of non-linear four wave interactions in discrete spectral wave models, Coastal Eng. 53, 223-242.

[54] WAMDI Group (1988), The WAM model - a third generation ocean wave prediction model. J. of Phys. Oceanog., 18, 1775-1810.

[55] Webb, D.J. (1978), Nonlinear transfers between sea waves, Deep Sea Res. 25, 279-298. 
[56] Zakharov, V.E. (1968), Stability of periodic waves of finite amplitude on the surface of a deep fluid, J. Appl. Mech. Tech. Phys. (English translation) 2, 190-1904.

[57] Zakharov, V.E., V.S. L'vov, V. S., and G. Falkovich (1992), Kolmogorov Spectra of Turbulence, Springer.

[58] Zijlema, M., Stelling, G.S. and Smit P. (2011), SWASH: An operational public domain code for simulating wave fields and rapidly varied flows in coastal waters. Coastal Engineering , 58: 992-1012. 

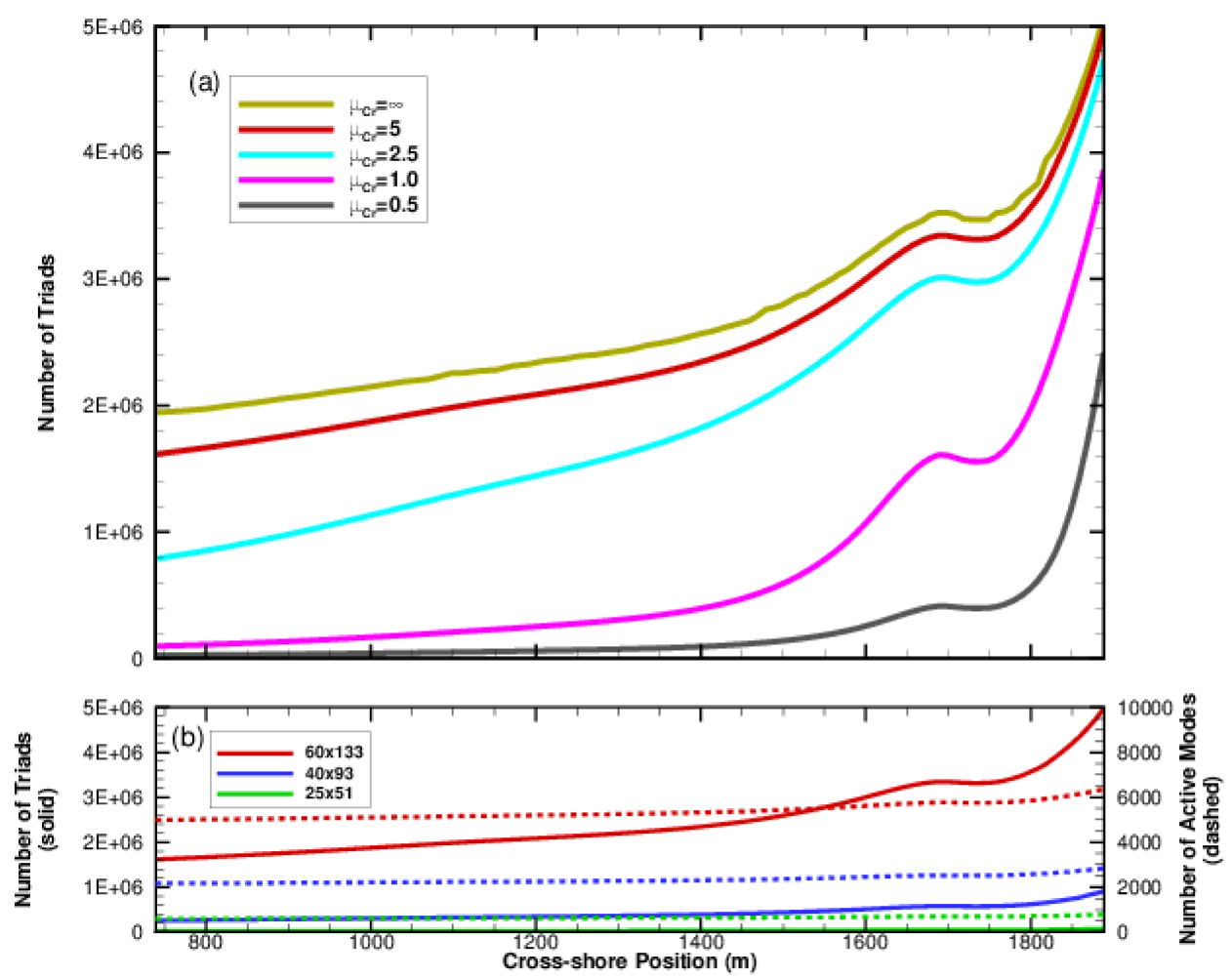

Figure 1: The dependency of the number of triads and active modes on the cross-shore position and the cut-off detuming parameter $\mu_{C r}$. a) The number of triads for several values of $\mu_{C r}$ and (b) the number of active modes and triads for the different domain sizes (see also the Appendix) using $\mu_{C r}=5$. 

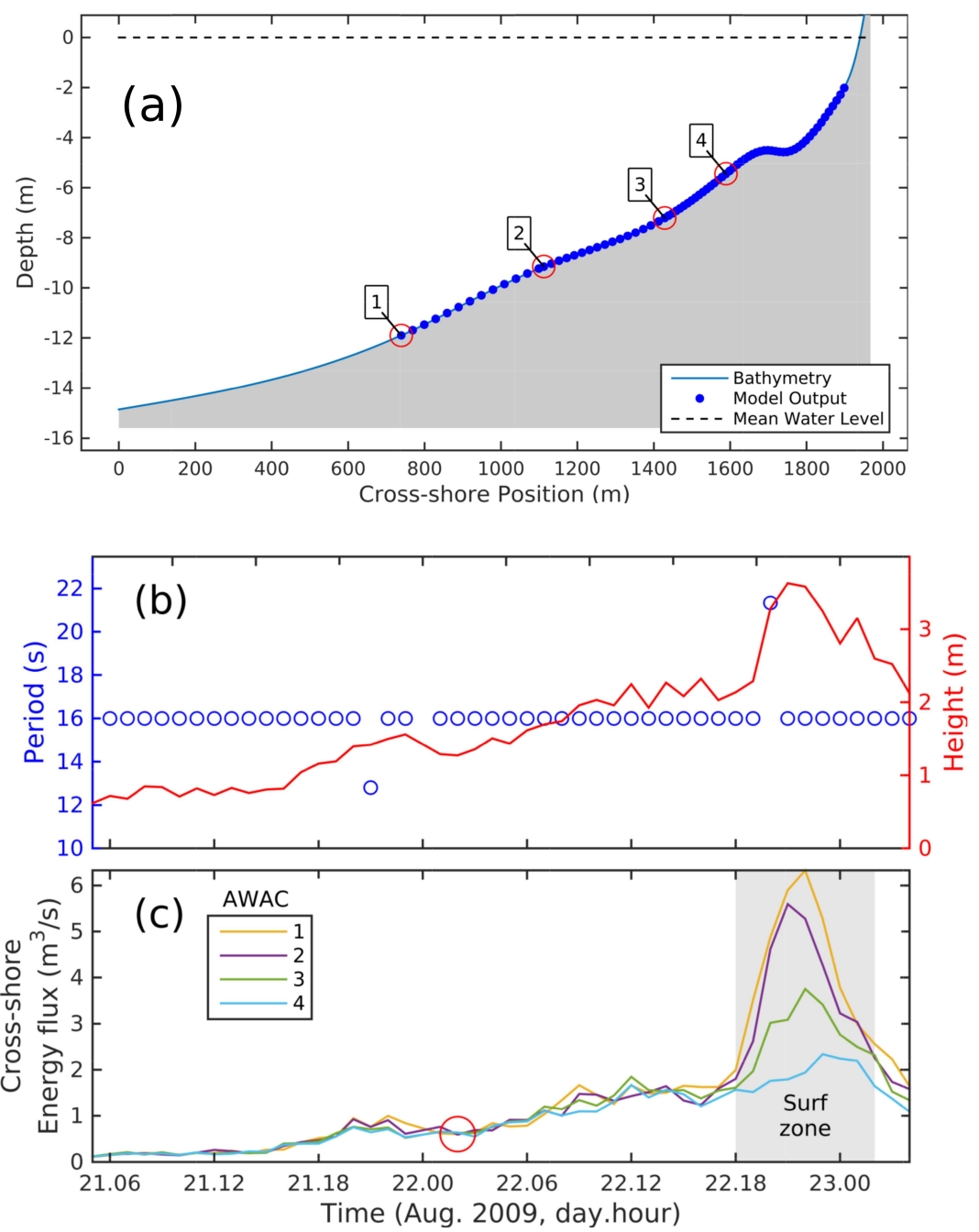

Figure 2: a) Bathymetric profile of the nearshore zone at Duck, NC, with the locations of the USACE AWAC sensors ( 1 to 4, red circles), and model output (blue points). b) Peak period and significant height of waves recorder at AWAC 1 (panel a) during Hurricane Bill. c) Estimate of cross-shore energy flux at the location of the AWAC sensors. The array is in the shoaling zone between Aug. 21st 5:00 hr and Aug. 22nd 18:00 hr, and in the surf zone during the peak of the storm (gray rectangle, excluded). The red circle marks the data set discussed in this study. 

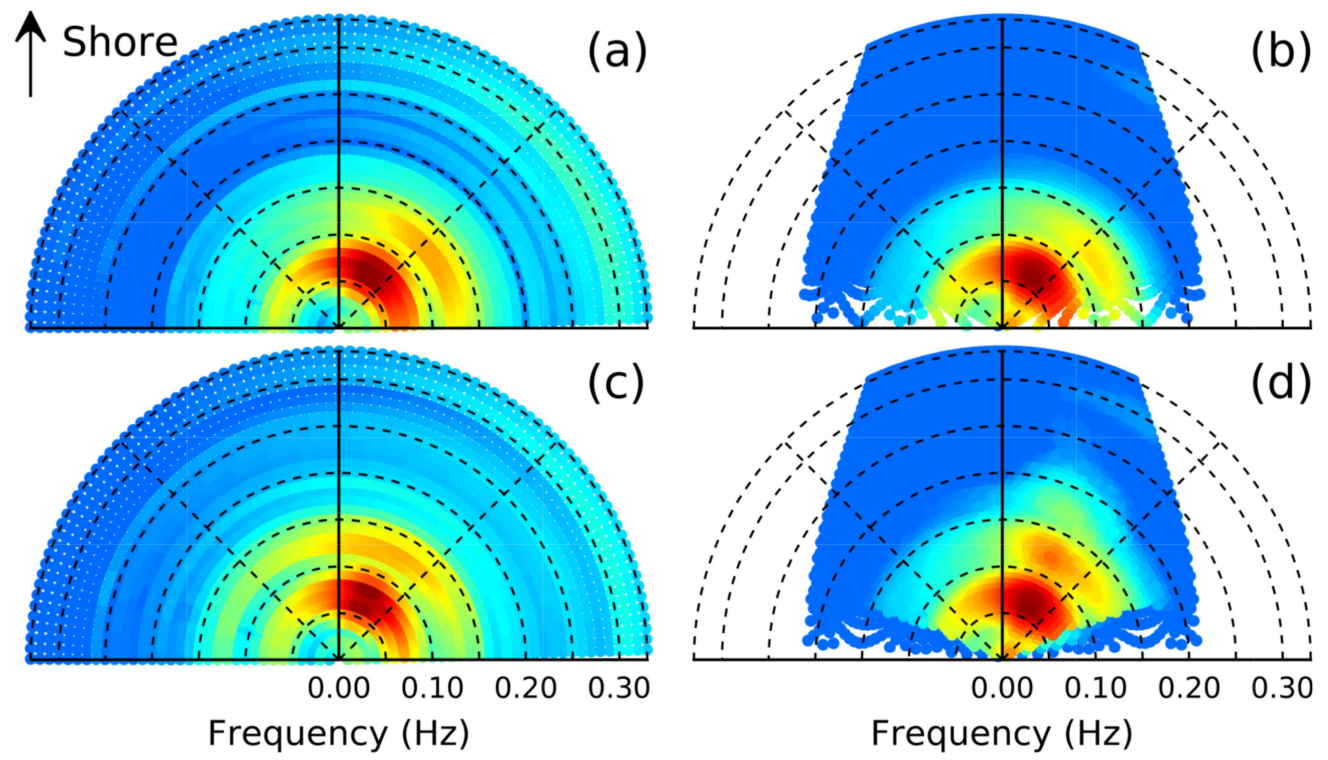

Figure 3: Comparison of observed (AWAC, left) and numerically simulated (right) cross-shore evolution of directional spectrum on August 22nd, 02:00 hr. a-b) Location of AWAC 1, 11.3-m depth (initial point); c-d) location of AWAC 2, $8.6-\mathrm{m}$ depth. Spectra are normalized by peak value; color scale is logarithmic.
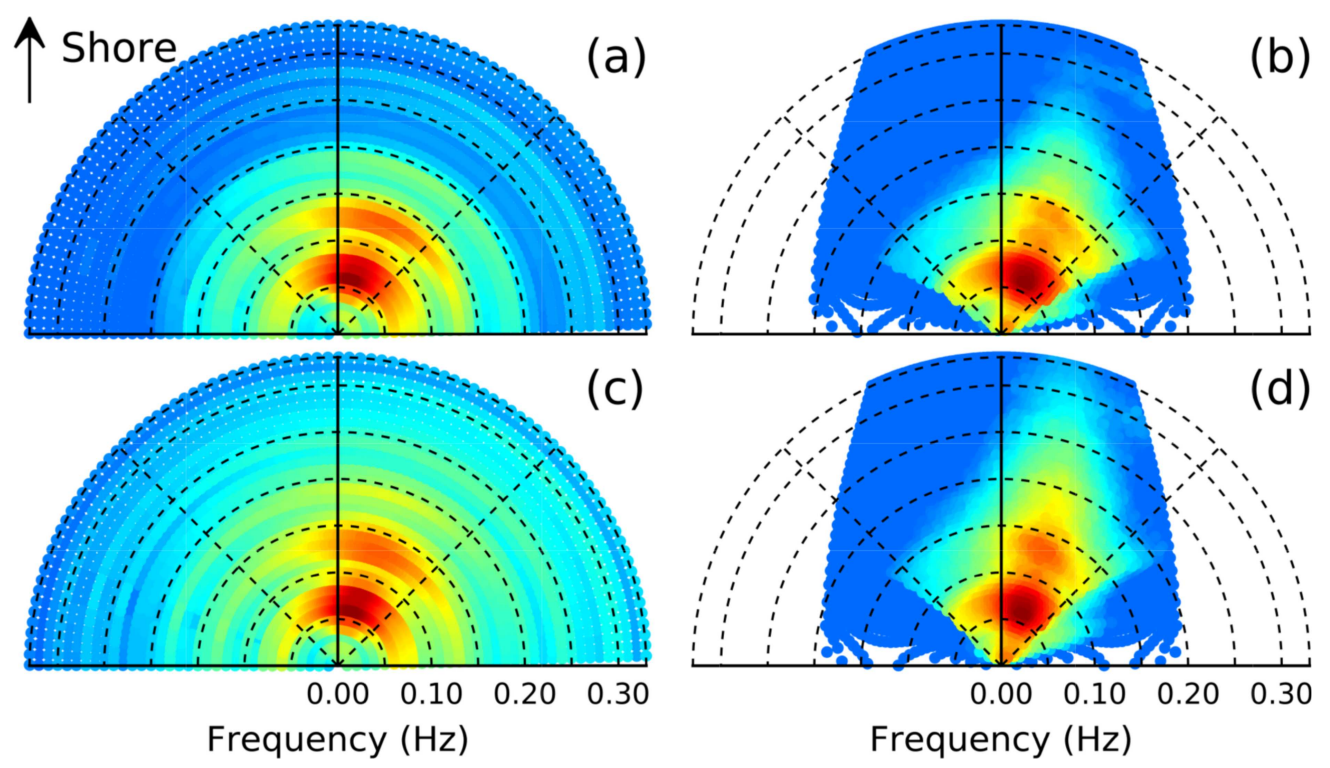

Figure 4: Comparison of observed (AWAC, left) and numerically simulated (right) cross-shore evolution of directional spectrum on August 22nd, 02:00 hr (continued). a-b) Location of AWAC 3, 6.6-m depth (initial point); c-d) location of AWAC 4, 4.9-m depth. Spectra are normalized by peak value; color scale is logarithmic. 


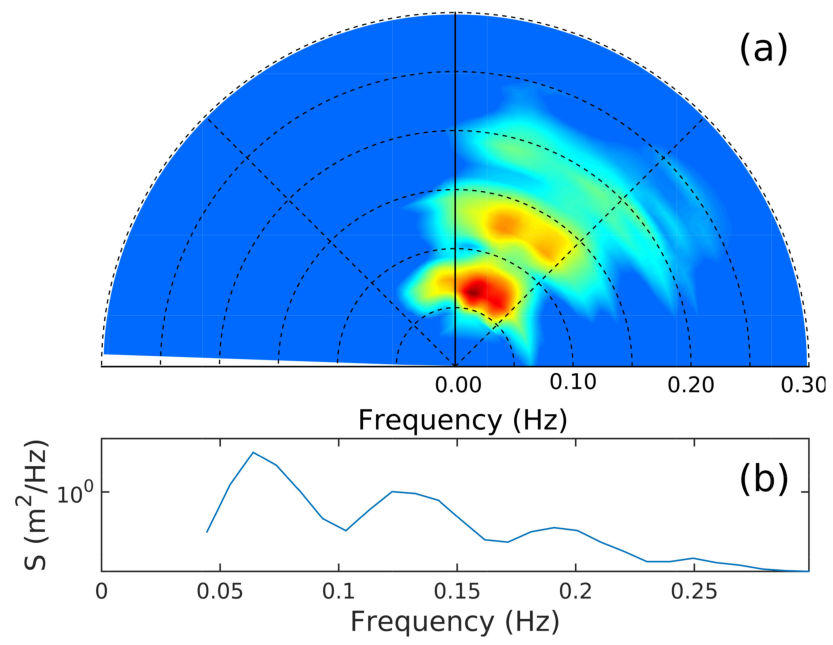

Figure 5: a) Directional spectrum August 22nd, 02:00 hr estimated based on the FRF 8-m array observations. Spectra are normalized by peak value; color scale is logarithmic. Compare with Figure 3c). b) Frequency spectrum.
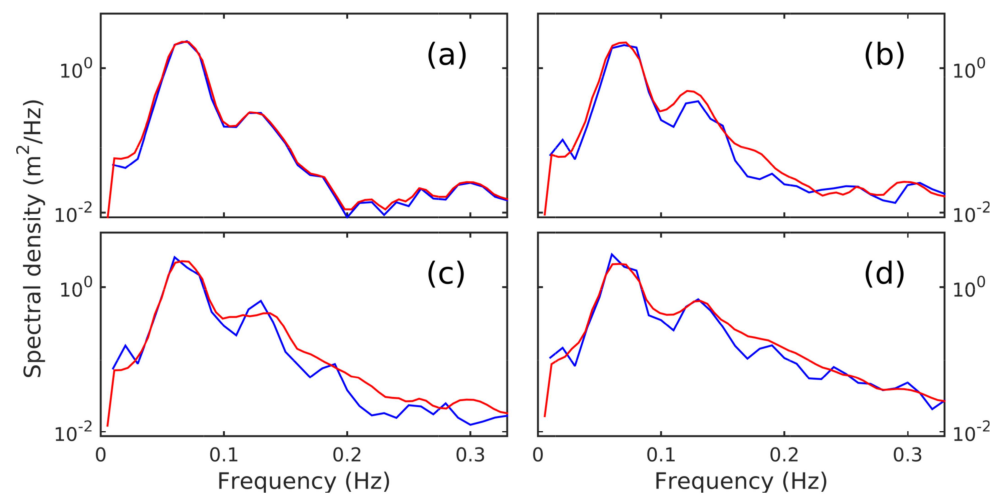

Figure 6: Cross-shore evolution of frequency spectrum on August 22nd, 02:00 hr; blue - AWAC estimates, red numerical model. a) AWAC 1; b) AWAC 2; c) AWAC 3; d) AWAC 4.
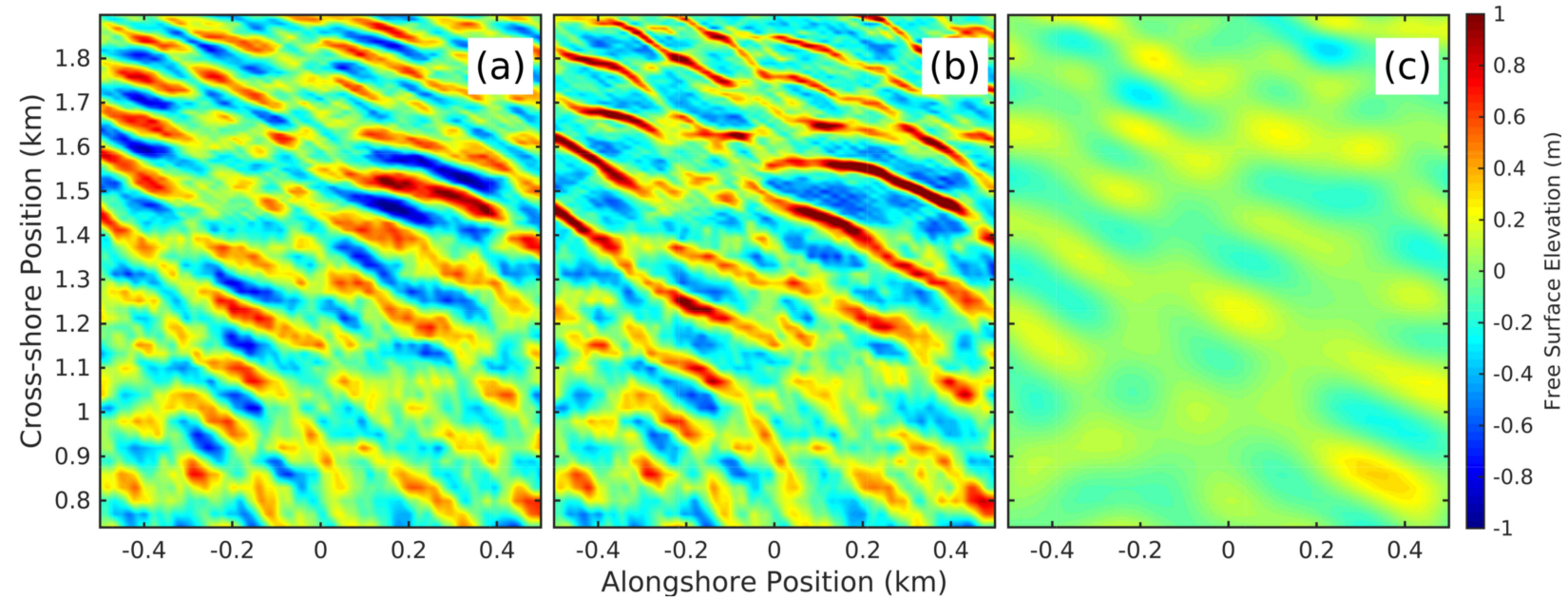

Figure 7: An example of one realization of the numerically-simulated free surface elevation. a) linear evolution; b) nonlinear evolution (TRIADS); c) corresponding nonlinear IG wave field. 


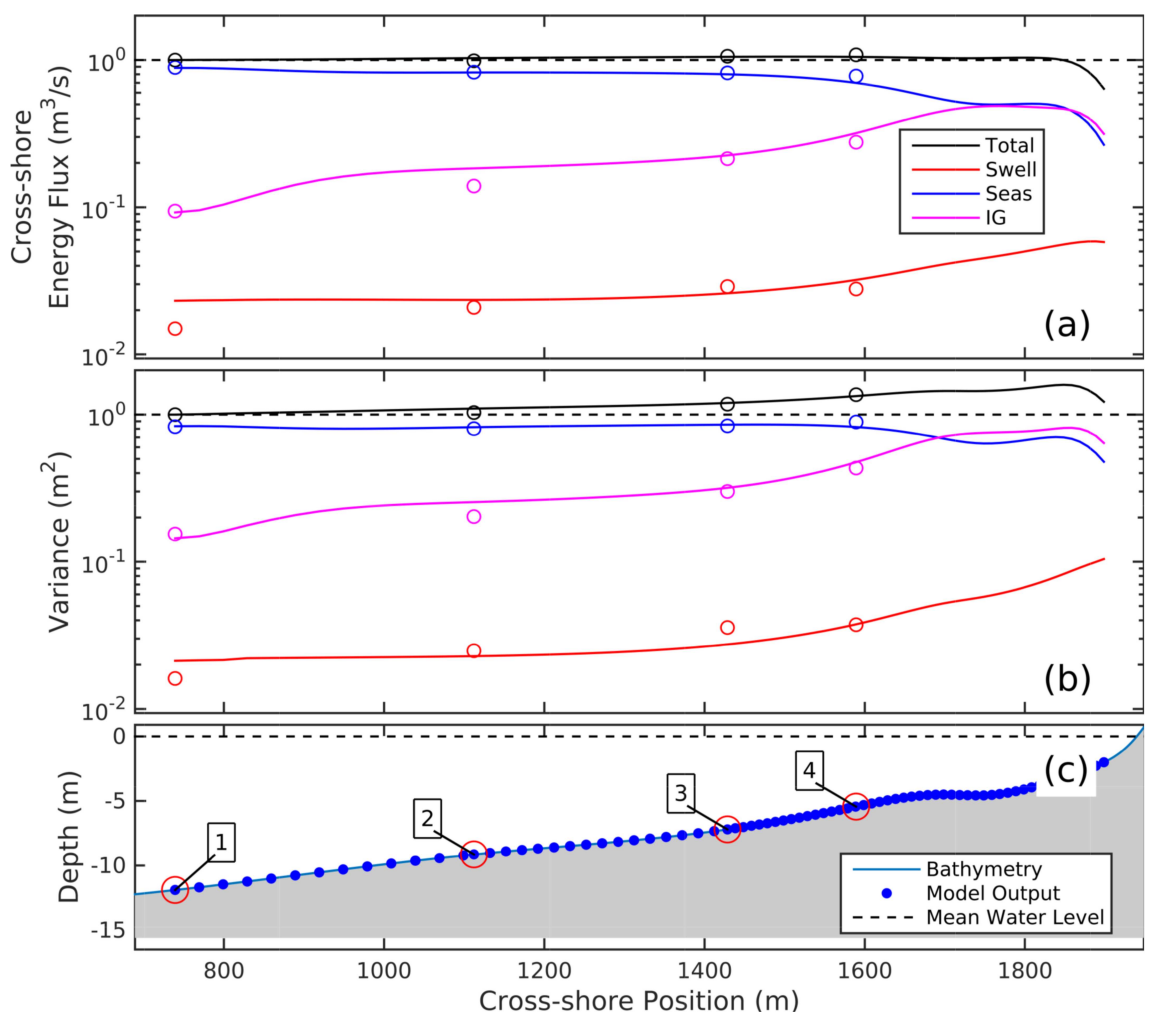

Figure 8: Comparison of observed (circles) and simulated (lines) shoaling transformation of band-integrated a) crossshore energy flux, normalized by the total cross-shore flux estimated at AWAC 1, and b) variance. The frequency bands are defined as: IG band: $f<0.5 f_{\text {Peak }}$; swell band: $0.5 f_{\text {Peak }} \leq f<1.5 f_{\text {Peak }}$; seas band: $f \geq 1.5 f_{\text {Peak }}$; where $f$ is the frequency, and $f_{\text {Peak }}$ is the frequency of the spectral peak. c) Bathymetric profile. 


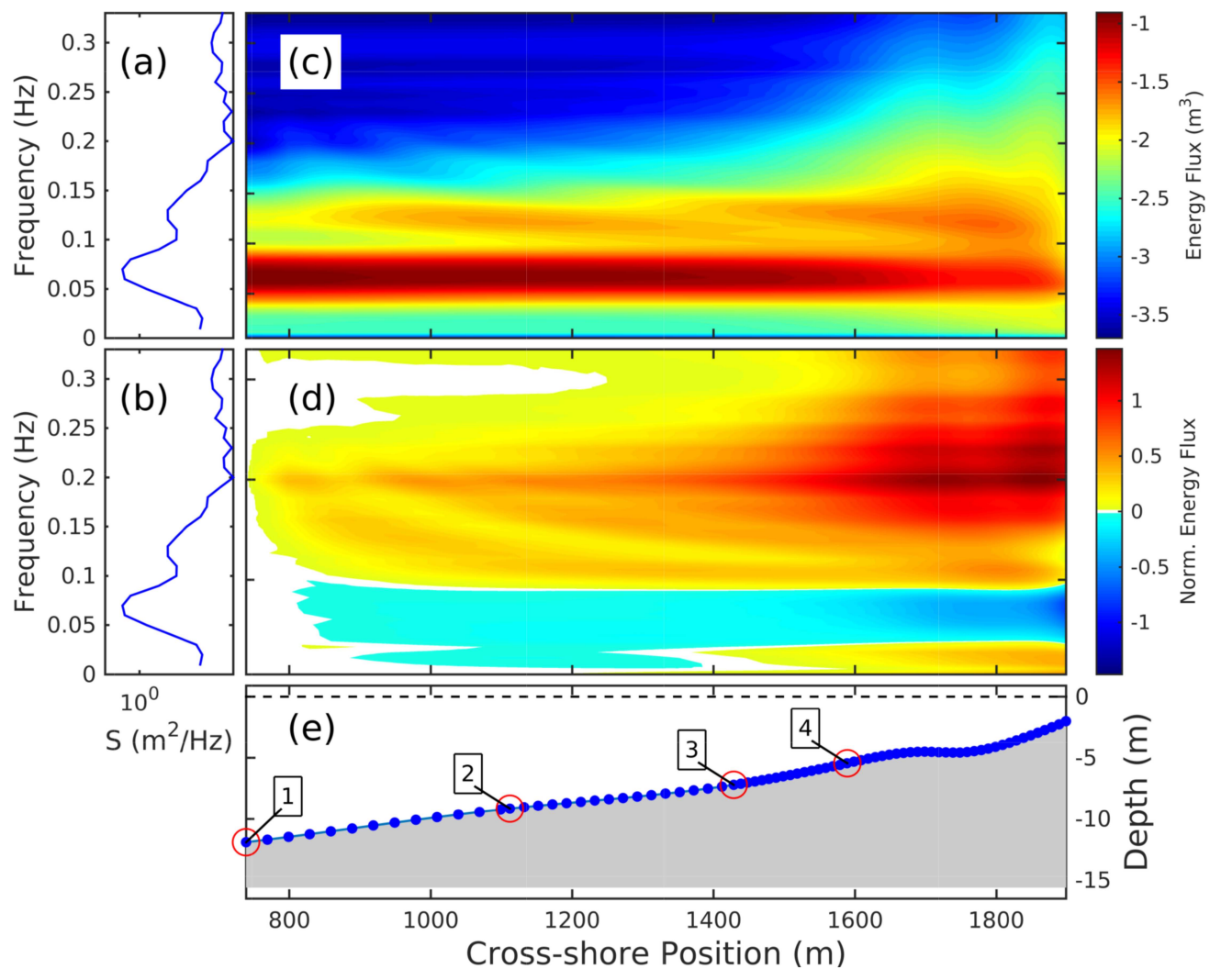

Figure 9: Shoaling transformation of the cross-shore energy flux, numerical simulations. a-b) Initial frequency spectrum; c) Evolution of the modal cross-shore energy flux; d) Amplification factor of the modal cross-shore energy flux (modal flux normalized by the value at the initial point, log scale). The amplification factor for a linear model is 1 for all modes. e) Bathymetric profile. 


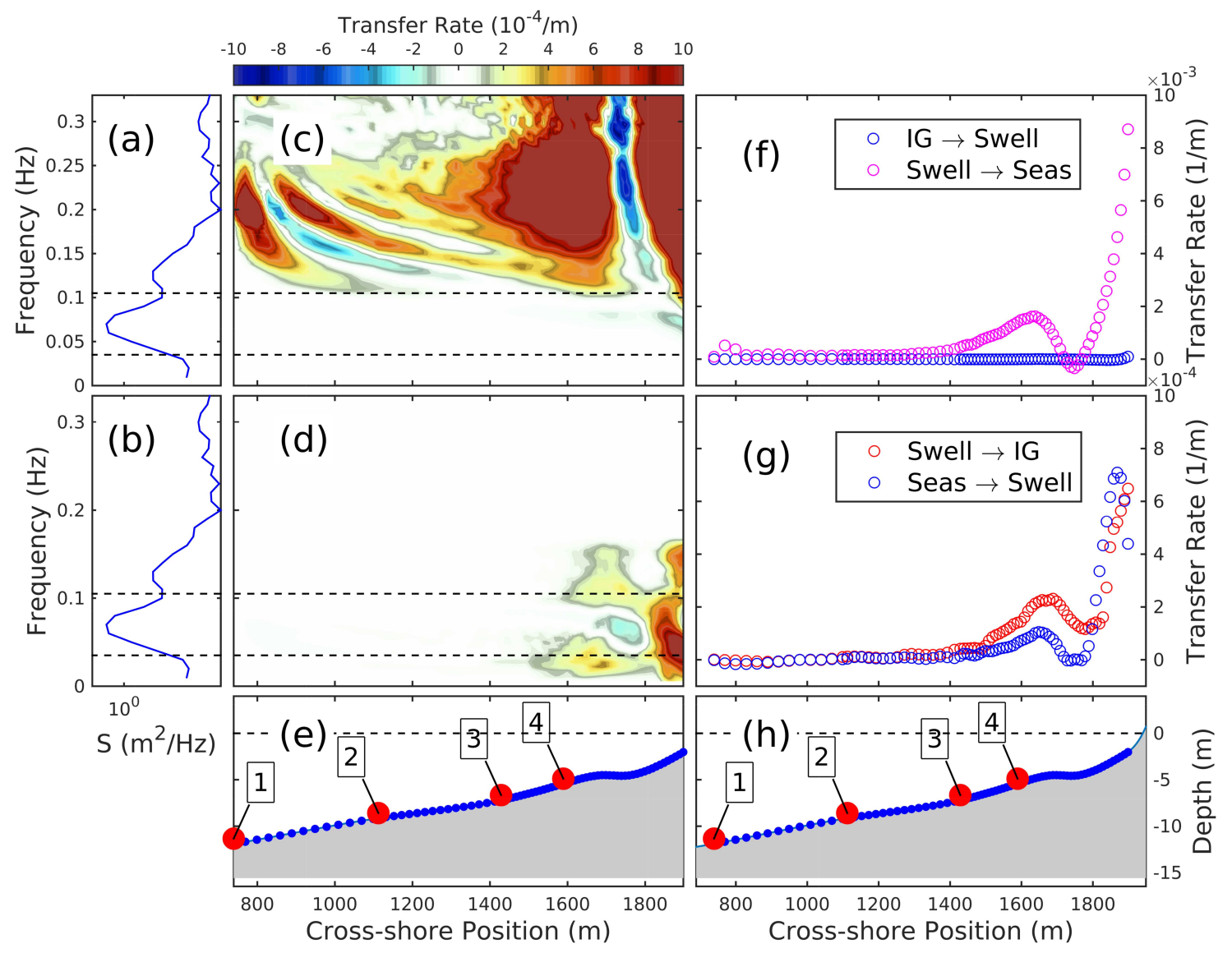

Figure 10: Numerical simulations: nonlinear transfer rates averaged over realizations and directional bands. a,b) Initial frequency spectrum. Frequency distribution of transfer rates associated with c) sum interactions, and d) difference interactions (equation 18). For a given frequency, panel c shows the total gain/loss from interactions with lower frequencies; panel d shows total gain/loss from interactions with higher frequencies. The frequency bands corresponding to IG, swell and seas are separated by dashed lines. Positive values represent growth. Band averages of transfer rates associated with f) sum interactions, and g) difference interactions (circles: data; line: smoothed trend). e,h) Bathymetric profile. In the legends in panels $\mathrm{f}-\mathrm{g}$, arrows indicate the direction of positive tra5sfer rates. 


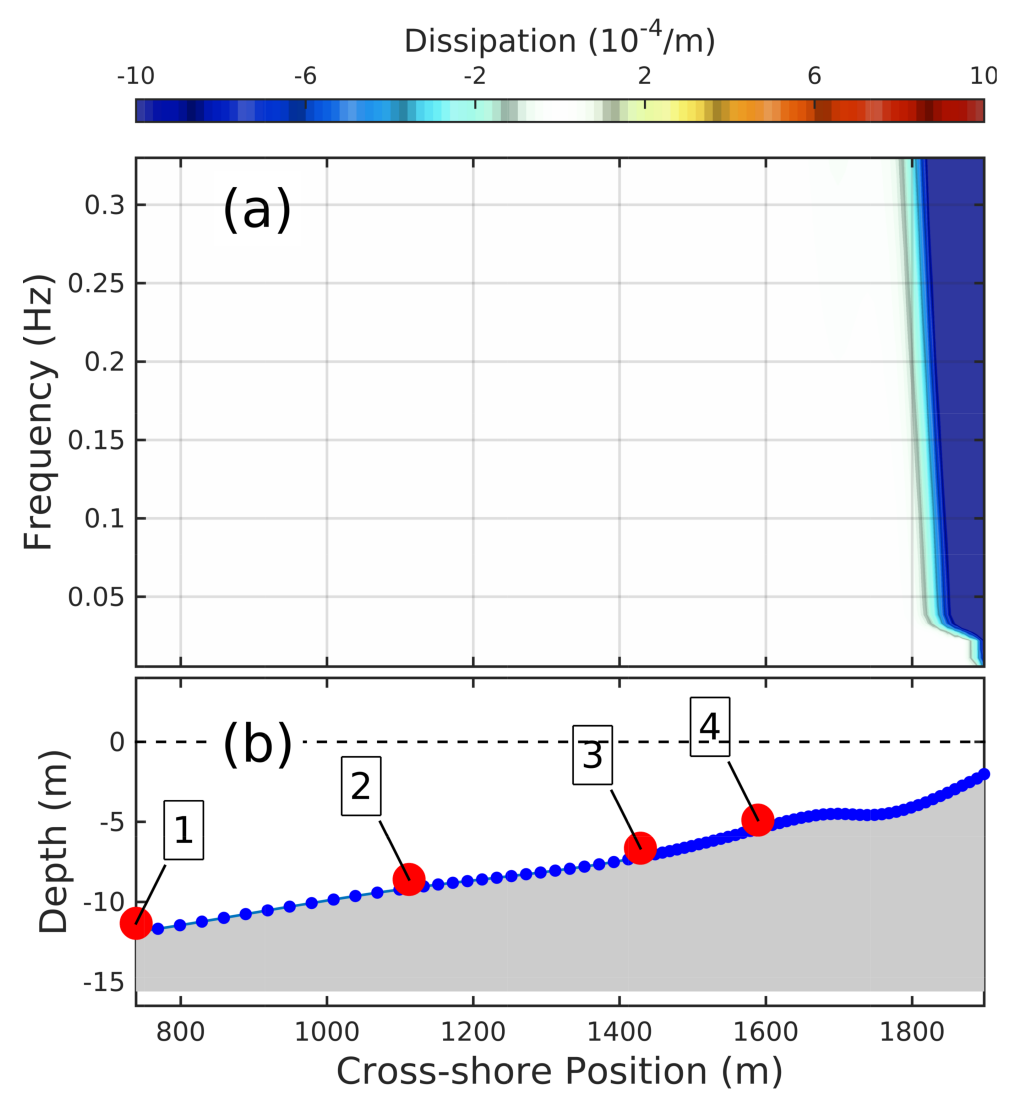

Figure 11: Numerical simulations: a) spectral breaking-induced dissipation rates averaged over realizations and directional bands. Positive values represent growth. b) Bathymetric profile.

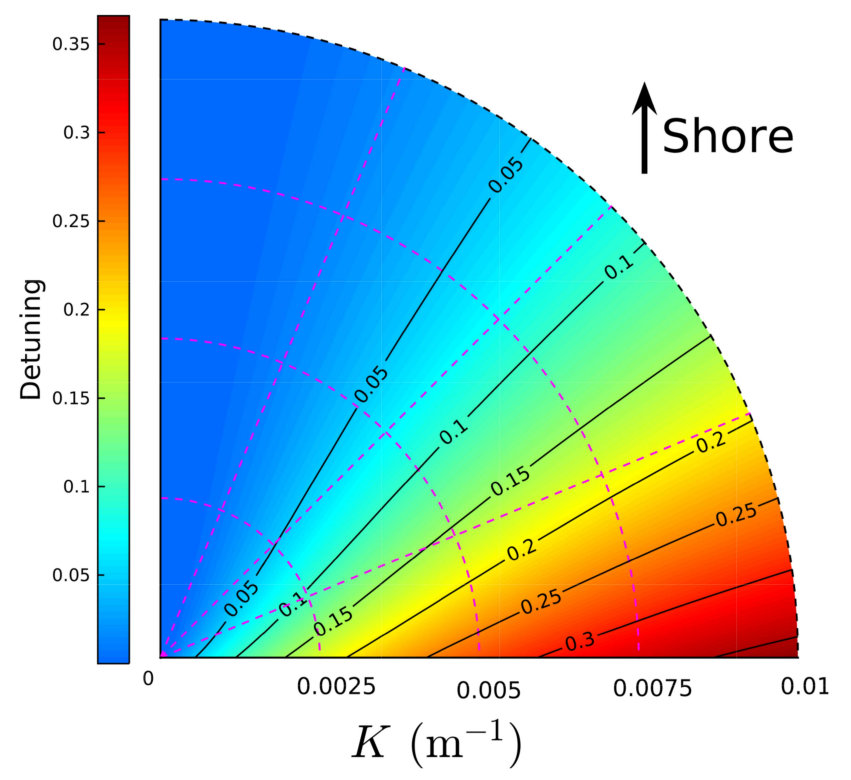

Figure 12: The dependency of the detuning parameter $\mu$ (equation 20) for different values of the angle $\alpha_{P Q}$. Mode $Q$ is propagating perpendicular to the shoreline $\left(\alpha_{Q}=0 ; \kappa_{Q}=0\right)$, and $K_{Q}=0.01 \mathrm{~m}^{-1}$. The detuning parameter $\mu=\mu\left(\alpha_{P}, K_{P}\right)$ is represented here for $0<\alpha_{P}<\frac{\pi}{2}$ and $0<K_{P}<0.01 \mathrm{~m}^{-1}$. 

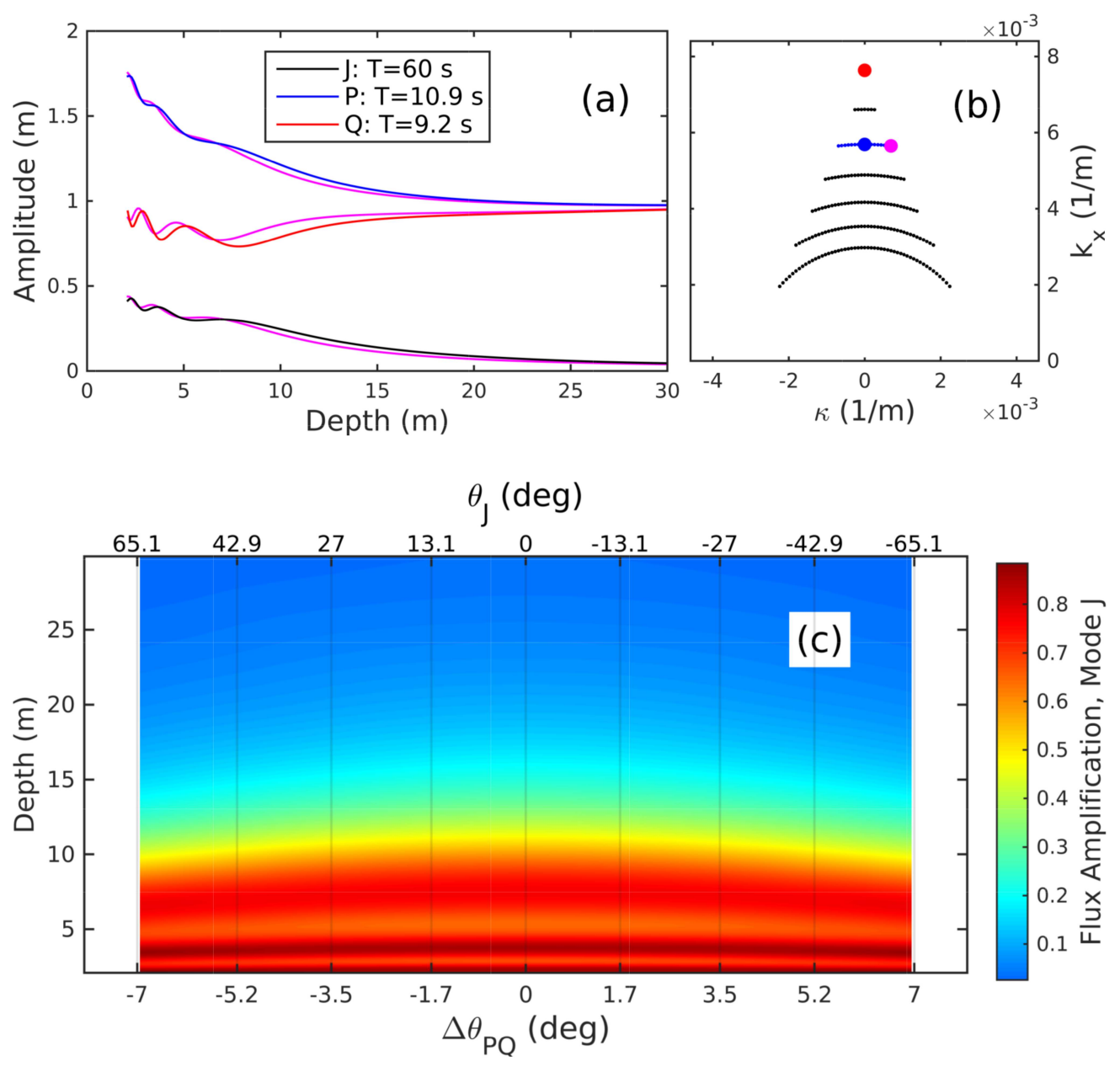

Figure 13: Dependency on directional spread of interaction efficiency for a triad $J=Q-P$ (equation 7) propagating over a plane beach of 0.01 slope, with mode $Q$ perpendicular to the shoreline. Initial amplitudes are $a_{Q}=a_{P}=1 \mathrm{~m}$; $a_{J}$ is computed as a bound wave at the initial point of integration in 50-m depth. a) Evolution of amplitudes. Blue, red, and black lines correspond to modes $P$ and $Q$ nearly parallel (dots blue and red, panel b). Magenta lines correspond to largest angular separation between $P$ and $Q$ (red and magenta dots in panel b). b) Wave-number grid (black dots) corresponding to an equally spaced $f-\kappa$ (equation 2) covering the domain of leaky difference triads at initial point (50-m depth). Red dot is mode $Q$. c) Flux amplification (cross-shore energy flux normalized by initial flux) as a function of the cross-shore position and angular separation between modes $P$ and $Q$. a 

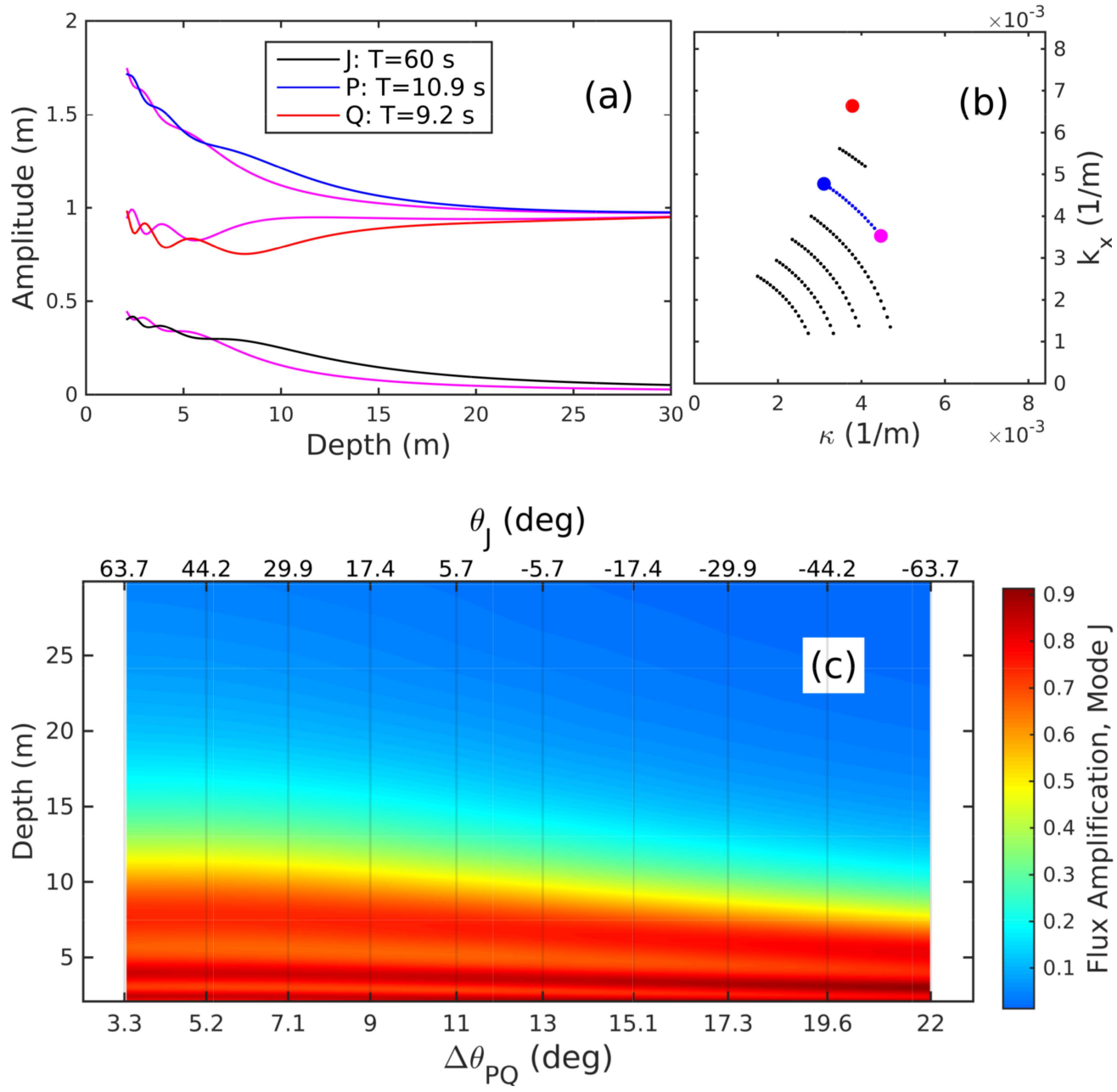

Figure 14: Same as Figure 13 but for model $Q$ propagating at 30 degrees with respect to the shore-normal direction. 


\begin{tabular}{|c|c|c|}
\cline { 2 - 3 } \multicolumn{1}{c|}{} & Dell Optiplex 9020 & Dell Precision T7610 \\
\hline CPU & One Intel i7-4770 @ 3.40 GHz & Two Xeon E5-2697 v2 @ 2.70 GHz \\
\hline Microarchitecture & Haswell & Ivy Bridge \\
\hline Cache & $8 \mathrm{MB}$ & $30 \mathrm{MB}$ \\
\hline Cores & 4 & 24 \\
\hline Memory & 16 GB (2x8GB) 1600 MHZ DDR3 & $128 \mathrm{~GB}$ (8x16GB) 1600 MHz DDR3 \\
\hline Storage & $500 \mathrm{~GB}$ Solid State Drive & 256 GB Solid State Drive \\
\hline Cost per Core (4/2014) & $\$ 239$ & $\$ 357$ \\
\hline
\end{tabular}

Table 1: Hardware characteristics of the two computational platforms used for model testing.

\begin{tabular}{|c|c|c|c|c|c|c|c|c|c|}
\hline & \multicolumn{3}{|c|}{ Dell Optiplex 9020} & \multicolumn{6}{|c|}{ Dell Precision T7610 } \\
\hline & \multicolumn{3}{|c|}{ Number of Cores } & \multicolumn{6}{|c|}{ Number of Cores } \\
\hline Domain & 1 & 2 & 3 & 1 & 2 & 4 & 8 & 16 & 24 \\
\hline & \multicolumn{9}{|c|}{ Total Solver Time per Realization (s) } \\
\hline $20 \times 51$ & 29.0 & 15.1 & 12.3 & 46.6 & 24.8 & 19.8 & 14.2 & 10.2 & 10.5 \\
\hline $40 \times 93$ & 370.6 & 189.0 & 148.4 & 584.8 & 294.8 & 230.2 & 147.4 & 87.5 & 61.9 \\
\hline $60 \times 133$ & 2074.5 & 1060.3 & 795.2 & 3315.2 & 1688.5 & 1261.0 & 785.9 & 439.7 & 311.6 \\
\hline & \multicolumn{9}{|c|}{ Parallel Speedup } \\
\hline $20 \times 51$ & 1.00 & 1.92 & 2.36 & 1.00 & 1.88 & 2.36 & 3.29 & 4.55 & 4.42 \\
\hline $40 \times 93$ & 1.00 & 1.96 & 2.50 & 1.00 & 1.98 & 2.54 & 3.97 & 6.68 & 9.45 \\
\hline $60 \times 133$ & 1.00 & 1.96 & 2.61 & 1.00 & 1.96 & 2.63 & 4.22 & 7.54 & 10.64 \\
\hline & \multicolumn{9}{|c|}{ Parallel Efficiency } \\
\hline $20 \times 51$ & $100.0 \%$ & $96.0 \%$ & $58.9 \%$ & $100.0 \%$ & $93.8 \%$ & $58.9 \%$ & $41.1 \%$ & $28.5 \%$ & $18.4 \%$ \\
\hline $40 \times 93$ & $100.0 \%$ & $98.0 \%$ & $62.4 \%$ & $100.0 \%$ & $99.2 \%$ & $63.5 \%$ & $49.6 \%$ & $41.8 \%$ & $39.4 \%$ \\
\hline $60 \times 133$ & $100.0 \%$ & $97.8 \%$ & $65.2 \%$ & $100.0 \%$ & $98.2 \%$ & $65.7 \%$ & $52.7 \%$ & $47.1 \%$ & $44.3 \%$ \\
\hline
\end{tabular}

Table 2: TRIADS performance for a single realization (here, a single RPA realization) of the OpenMP parallel version, for the two test platforms. 


\begin{tabular}{|c|c|c|c|c|c|c|c|c|c|}
\hline \multirow[b]{3}{*}{$\mathrm{D}$} & \multirow{2}{*}{\multicolumn{3}{|c|}{$\begin{array}{c}\text { Dell Optiplex } 9020 \\
\text { Number of Cores }\end{array}$}} & \multicolumn{6}{|c|}{ Dell Precision T7610 } \\
\hline & & & & \multicolumn{6}{|c|}{ Number of Cores } \\
\hline & 1 & 2 & 3 & 1 & 2 & 4 & 8 & 16 & 24 \\
\hline & \multicolumn{9}{|c|}{ Total Solver Time /Realization(s) } \\
\hline $20 \times 51$ & 29.6 & 29.9 & 32.5 & 46.9 & 46.1 & 47.1 & 47.1 & 47.9 & 49.8 \\
\hline $40 \times 93$ & 369.9 & 380.7 & 399.2 & 587.7 & 582.3 & 602.7 & 595.7 & 601.4 & 602.8 \\
\hline \multirow[t]{2}{*}{$60 \times 133$} & 2073.9 & 2134.3 & 2599.7 & 3335.8 & 3386.6 & 3382.8 & 3419.0 & 3430.8 & 3414.2 \\
\hline & \multicolumn{9}{|c|}{ Parallel Speedup } \\
\hline $20 \times 51$ & 1.00 & 1.98 & 3.65 & 1.00 & 2.03 & 3.99 & 7.96 & 15.66 & 22.60 \\
\hline $40 \times 93$ & 1.00 & 1.94 & 3.71 & 1.00 & 2.02 & 3.90 & 7.89 & 15.64 & 23.40 \\
\hline \multirow[t]{2}{*}{$60 \times 133$} & 1.00 & 1.94 & 3.19 & 1.00 & 1.97 & 3.94 & 7.81 & 15.56 & 23.45 \\
\hline & \multicolumn{9}{|c|}{ Parallel Efficiency } \\
\hline $20 \times 51$ & $100.0 \%$ & $98.8 \%$ & $91.2 \%$ & $100.0 \%$ & $101.7 \%$ & $99.7 \%$ & $99.6 \%$ & $97.9 \%$ & $94.2 \%$ \\
\hline $40 \times 93$ & $100.0 \%$ & $97.1 \%$ & $92.7 \%$ & $100.0 \%$ & $100.9 \%$ & $97.5 \%$ & $98.7 \%$ & $97.7 \%$ & $97.5 \%$ \\
\hline $60 \times 133$ & $100.0 \%$ & $97.2 \%$ & $79.8 \%$ & $100.0 \%$ & $98.5 \%$ & $98.6 \%$ & $97.6 \%$ & $97.2 \%$ & $97.7 \%$ \\
\hline
\end{tabular}

Table 3: TRIADS performance for multiple realizations (here, a single RPA realization) of single-processor runs, for the two test platforms.

\begin{tabular}{|c|c|c|c|c|}
\hline & & \multicolumn{3}{|c|}{ Domain } \\
\hline & & $20 \times 51$ & $40 \times 93$ & $60 \times 133$ \\
\hline \multirow{10}{*}{ Optiplex } & Total Number of Modes & 1020 & 3720 & 7980 \\
\hline & Number of Active Modes $\left(\mu_{C r}=5\right)$ & 782 & 2840 & 6358 \\
\hline & Normalized Number of Active Modes $\left(\mu_{C r}=5\right)$ & 1.0 & 3.6 & 8.1 \\
\hline & Number of Triads $\left(\mu_{C r}=5\right)$ & 67982 & 908836 & 4975510 \\
\hline & Normalized Number of Triads $\left(\mu_{C r}=5\right)$ & 1.0 & 13.4 & 73.2 \\
\hline & Single Core CPU Time(s/core) & 29.6 & 369.9 & 2073.9 \\
\hline & Normalized Single Core CPU Time & 1.0 & 12.5 & 70.1 \\
\hline & Time per Active Mode (s/mode) & 0.04 & 0.13 & 0.33 \\
\hline & Time per Triad (s/triad) & 0.000435 & 0.000407 & 0.000417 \\
\hline & (Cost per Core) per (Time per Triad) (\$ triad / core s) & 548994 & 587423 & 573528 \\
\hline \multirow{5}{*}{ Precision } & Single Core CPU Time(s/core) & 46.9 & 587.7 & 3315.2 \\
\hline & Normalized Single Core CPU Time & 1.0 & 12.5 & 70.7 \\
\hline & Time per Active Mode (s/mode) & 0.06 & 0.21 & 0.52 \\
\hline & Time per Triad (s/triad) & 0.000690 & 0.000647 & 0.000666 \\
\hline & (Cost per Core) per (Time per Triad) (\$ triad / cores) & 346459 & 369687 & 358795 \\
\hline \multirow{2}{*}{$\begin{array}{l}\text { Optiplex/ } \\
\text { Precision }\end{array}$} & Single Core CPU Time(s/core) & 0.63 & 0.63 & 0.63 \\
\hline & (Cost per Core) per (Time per Triad) (\$ triad / cores) & 1.58 & 1.59 & 1.60 \\
\hline
\end{tabular}

Table 4: Relative performance and cost effectiveness of the two test platforms. 